\title{
EXISTENCE AND NON-EXISTENCE OF TRANSITION FRONTS FOR BISTABLE AND IGNITION REACTIONS
}

\author{
ANDREJ ZLATOŠ
}

\begin{abstract}
We study reaction-diffusion equations in one spatial dimension and with general (space- or time-) inhomogeneous mixed bistable-ignition reactions. For those satisfying a simple quantitative hypothesis, we prove existence and uniqueness of transition fronts, as well as convergence of "typical" solutions to the unique transition front (the existence part even extends to mixed bistable-ignition-monostable reactions). These results also hold for all pure ignition reactions without any other hypotheses, but not for all pure bistable reactions. In fact, we find examples of either spatially or temporally periodic pure bistable reactions (independent of the other space-time variable) for which we can prove non-existence of transition fronts. These are the first such results for periodic media which are non-degenerate in a natural sense, and the spatially periodic examples also prove a conjecture from [7].
\end{abstract}

\section{INTRODUCTION}

We study reaction-diffusion equations

$$
u_{t}=u_{x x}+f(x, u)
$$

and

$$
u_{t}=u_{x x}+f(t, u)
$$

in one spatial dimension. These equations are used to model a host of natural processes such as combustion, population dynamics, pulse propagation in neural networks, or solidification dynamics. We will consider here the cases of either spatially (1.1) or temporally (1.2) inhomogeneous mixed bistable-ignition reactions. We are primarily interested in general (non-periodic) reactions, but our results are new even in the periodic case.

For homogeneous media, one usually considers bistable reactions to have $\tilde{\theta} \in(0,1)$ such that $f(0)=f(\tilde{\theta})=f(1)=0$, with $f<0$ on $(0, \tilde{\theta})$ and $f>0$ on $(\tilde{\theta}, 1)$, while ignition reactions have $f=0$ on $(0, \tilde{\theta})$ and $f>0$ on $(\tilde{\theta}, 1)$. It is also standard to consider $f$ non-increasing near 0 and 1 (and sometimes even $f^{\prime}(1)<0$, along with $f^{\prime}(0)<0$ for bistable $f$ ). One is then interested in solutions $0 \leq u \leq 1$ which transition between the (stable) equilibria $u \equiv 0$ and $u \equiv 1$, modeling invasions of one equilibrium of the relevant physical process by another. Typically these include solutions evolving from initial data which are spark-like (with $\lim _{|x| \rightarrow \infty} u(0, x)=0$ ), or front-like (with $\lim _{x \rightarrow \infty} u(0, x)=0$ and $\left.\liminf _{x \rightarrow-\infty} u(0, x)>\tilde{\theta}\right)$. It is customary to also assume $\int_{0}^{1} f(u) d u>0$, so that solutions which are initially above some $\beta>\tilde{\theta}$ on a large enough $\beta$-dependent interval converge locally uniformly to 1 as $t \rightarrow \infty$ (i.e., they propagate). One is then interested in the nature of the transition from 0 to 1 . (Note that the roles of 0 and 1 are reversed if $\int_{0}^{1} f(u) d u<0$ for bistable $f$.)

The study of transitions between equilibria of reaction-diffusion equations has seen a lot of activity since the seminal papers of Kolmogorov, Petrovskii, Piskunov [15] and Fisher [13] 
(who studied homogeneous reactions). We are here interested in this question for $f$ which also depends on $x$ or $t$, and we will also relax the requirement for a single sign change of $f(x, \cdot)$ or $f(t, \cdot)$ in $(0,1)$. We will therefore assume the following hypothesis. Let us consider only (1.1) for the time being; (1.2) will be treated afterwards.

Hypothesis (H): $f$ is Lipschitz with constant $K \geq 1$,

$$
f(x, 0)=f(x, 1)=0 \quad \text { for } x \in \mathbb{R},
$$

and there is $\theta>0$ such that for each $x \in \mathbb{R}, f$ is non-increasing in $u$ on $[0, \theta]$ and on $[1-\theta, 1]$. Moreover, there are $0<\theta_{1} \leq \theta_{0}<1$ and Lipschitz functions $f_{0}, f_{1}:[0,1] \rightarrow \mathbb{R}$ with $f_{0} \leq f_{1}$,

$$
\begin{gathered}
f_{0}(0)=f_{0}(1)=f_{1}(0)=f_{1}(1)=0, \\
f_{0} \leq 0 \text { on }\left(0, \theta_{0}\right) \quad \text { and } \quad f_{0}>0 \text { on }\left(\theta_{0}, 1\right), \\
f_{1} \leq 0 \text { on }\left(0, \theta_{1}\right) \quad \text { and } \quad f_{1}>0 \text { on }\left(\theta_{1}, 1\right), \\
\int_{0}^{1} f_{0}(u) d u>0,
\end{gathered}
$$

such that

$$
f_{0}(u) \leq f(x, u) \leq f_{1}(u) \quad \text { for }(x, u) \in \mathbb{R} \times[0,1]
$$

Definition 1.1. (i) We call any $f$ satisfying $(\mathrm{H})$ a $B I$ reaction (i.e., bistable-ignition).

(ii) If $f$ is a BI reaction and $f_{1}<0$ on $\left(0, \theta_{1}\right)$, then $f$ is a bistable reaction. If there is also an increasing function $\gamma:[0, \infty) \rightarrow[0, \infty)$ and for each $x \in \mathbb{R}$ there is $\tilde{\theta}_{x} \in\left[\theta_{1}, \theta_{0}\right]$ such that

$$
\operatorname{sgn}\left(u-\tilde{\theta}_{x}\right) f(x, u) \geq \gamma\left(\operatorname{dist}\left(u,\left\{0, \tilde{\theta}_{x}, 1\right\}\right)\right)
$$

for $u \in[0,1]$, then $f$ is a pure bistable reaction.

(iii) If $f$ is a $\mathrm{BI}$ reaction and $f_{0}=0$ on $\left(0, \theta_{0}\right)$, then $f$ is an ignition reaction. If there are also $\gamma$ and $\tilde{\theta}_{x}$ as in (ii) such that now $f(x, u)=0$ for $u \in\left[0, \tilde{\theta}_{x}\right]$ and

$$
f(x, u) \geq \gamma\left(\operatorname{dist}\left(u,\left\{\tilde{\theta}_{x}, 1\right\}\right)\right)
$$

for $u \in\left[\tilde{\theta}_{x}, 1\right]$, then $f$ is a pure ignition reaction.

Remark. We note that if instead $\theta=0=\theta_{1}$ in $(\mathrm{H})$, then $f$ is a mixed bistable-ignitionmonostable reaction, and it is a pure monostable reaction if (iii) above holds with $\tilde{\theta}_{x} \equiv 0$.

Let us now briefly review some of the relevant literature for bistable and ignition reactions in one dimension (their mixtures, allowed here, may not have been studied before). In these papers, (1.4) need not always be assumed for bistable reactions and other hypotheses may be included. There is also a large body of work on monostable reactions in one dimension, as well as on all reaction types in several dimensions, and the interested reader can consult [3, 32, 33] for reviews of these results and other related developments.

A useful tool in the study of the evolution of solutions of reaction-diffusion equations can often be special solutions called transition fronts. These are entire solutions $w$ of (1.1), defined in [18,25] for some special situations and later in [6] in more generality, satisfying

$$
\lim _{x \rightarrow-\infty} w\left(t, x+x_{t}\right)=1 \quad \text { and } \quad \lim _{x \rightarrow \infty} w\left(t, x+x_{t}\right)=0
$$


uniformly in $t \in \mathbb{R}$, with $x_{t}:=\max \left\{x \in \mathbb{R} \mid w(t, x)=\frac{1}{2}\right\}$ (which includes existence of $x_{t}$ ). This is the definition of the right-moving transition front, while the left-moving one is defined with 1 and 0 swapped. Note that we consider here only fronts connecting 0 and 1 , not those connecting other equilibria of the PDE (if they exist). Also, we will only consider here fronts with $0 \leq w \leq 1$, since we do not assume anything about $f(x, u)$ for $u \notin[0,1]$. It is easy to show, however, that if $f(x, u) \geq 0$ for $u<0$ and $f(x, u) \leq 0$ for $u>1$, then any transition front satisfies $0<w<1$ [6, 35]. Finally, we note that the definition we use here is that of transition fronts with a single interface. The definition in [6], restricted to one dimension, allows any bounded-in-time number of interfaces, but most papers studying transition fronts in one dimension use the (very natural) single interface version.

In media where there exists a unique right-moving and a unique left-moving transition front (up to a translation in $t$ ), one can sometimes show that typical solutions converge to their time-shifts as $t \rightarrow \infty$. The simplest such case are homogeneous media $f(x, u)=f(u)$, where transition fronts are known to be unique for ignition and bistable reactions, and take the form of traveling fronts $w(t, x)=W(x-c t)$ (right-moving) and $w(t, x)=W(-x-c t)$ (left-moving), with a unique front speed $c>0$ and the front profile $W$ solving $W^{\prime \prime}+c W^{\prime}+f(W)=0$ on $\mathbb{R}$ and having the limits $\lim _{s \rightarrow-\infty} W(s)=1$ and $\lim _{s \rightarrow \infty} W(s)=0$.

The situation is slightly more complicated for spatially periodic media, where existence and uniqueness of pulsating fronts (first defined in [29], these are transition fronts satisfying $u\left(t+\frac{p}{c}, x\right)=u(t, x-p)$ with $p$ the spatial period of $f$ and $c$ the front speed, whose profile is time-periodic in a moving frame) has been proved for fairly general ignition reactions [5] but only for some special cases of bistable reactions. This includes near-homogeneous reactions [30] (see also [31] for a related result), reactions with a constant $\tilde{\theta}_{x}$ (i.e., $\theta_{1}=\theta_{0}$ in $(\mathrm{H})$ ) [22], those for which (1.1) has no stable periodic steady states between 0 and 1 [10], and those with small or large spatial periods [7,8] (hence our Theorem 1.2)(i) below is new even for periodic bistable reactions). There is a good reason for such limitations: while uniqueness holds at least for non-stationary pulsating fronts if we also assume $f_{1}^{\prime}(0)<0$ and $f_{0}^{\prime}(1)<0$ [ , existence does not even for pure bistable reactions, as we show in Theorem 1.2(iii) below (and therefore also prove a conjecture from [7]).

Another reason for the added difficulties in the inhomogeneous bistable case is the fact that solutions may stop propagating and stationary fronts may exist, although not when (1.4) holds. This can naturally happen when $\int_{0}^{1} f(x, u) d u$ changes sign as $x$ varies [8], but it can even happen for periodic pure bistable reactions with $\int_{0}^{1} f(x, u) d u>0$ for all $x \in \mathbb{R}$. For instance, we can take $v(x):=\frac{1}{2}-\frac{1}{\pi} \arctan x$ and $g(u):=-v^{\prime \prime}\left(\tan \left(\frac{\pi}{2}-\pi u\right)\right)\left(\right.$ so that $v^{\prime \prime}+g(v)=$ 0 and $g$ is pure bistable with $\tilde{\theta}=\frac{1}{2}$ and $\left.\int_{0}^{1} g(u) d u=0\right)$. Then we take any $x$-periodic $f$ with $\int_{0}^{1} f(x, u) d u>0$ for each $x \in \mathbb{R}$ such that $f(x, u)=g(u)$ for $(x, u) \in \mathbb{R} \times\left(\left[0, \frac{1}{6}\right] \cup\left[\frac{1}{4}, \frac{3}{4}\right] \cup\left[\frac{5}{6}, 1\right]\right)$ as well as for $(x, u) \in(-\sqrt{3},-1) \times\left(\frac{3}{4}, \frac{5}{6}\right)$ and $(x, u) \in(1, \sqrt{3}) \times\left(\frac{1}{6}, \frac{1}{4}\right)$. Such pure bistable $f$ (not satisfying (1.4)) easily exists and satisfies $v^{\prime \prime}(x)+f(x, v(x))=0$ for all $x \in \mathbb{R}$ because $v((-\sqrt{3},-1))=\left(\frac{3}{4}, \frac{5}{6}\right)$ and $v((1, \sqrt{3}))=\left(\frac{1}{6}, \frac{1}{4}\right)$. We refer the reader to [8, 12, 16, 31] and the references therein for further studies of such wave-blocking phenomena for bistable reactions.

As for non-periodic media, it was proved in [19, 20, 22] for ignition reactions of the form $f(x, u)=a(x) g(u)$ with some bounded $a \geq 1$ and a pure ignition $g$ (in particular, $\theta_{1}=\theta_{0}$ ), that exponentially decaying front-like solutions converge to a unique right-moving front in 
$L^{\infty}(\mathbb{R})$ as $t \rightarrow \infty$, while spark-like ones converge to it in $L^{\infty}\left(\mathbb{R}^{+}\right)$and to a unique left-moving one in $L^{\infty}\left(\mathbb{R}^{-}\right)$. This was extended to general ignition reactions satisfying a non-vanishing condition in [34] (see Section 3 below). For bistable reactions, these results again hold for $f(x, u)=a(x) g(u)$ with $a \geq 1$ and a pure bistable $g$ (in particular, $\left.\theta_{1}=\theta_{0}\right)$ [22], and existence of transition fronts was proved earlier for general near-homogeneous bistable reactions in [30].

On the other hand, one can easily construct situations in which transition fronts (connecting 0 and 1) do not exist, even if $f$ satisfies $(\mathrm{H})$. A simple example is a homogeneous reaction with $f\left(\frac{1}{2}\right)=0$ which is bistable when restricted to $u \in\left[0, \frac{1}{2}\right]$ (with a unique front speed $c^{\prime}$ ) as well as when restricted to $u \in\left[\frac{1}{2}, 1\right]$ (with a unique front speed $c^{\prime \prime}$ ), and $f\left(u+\frac{1}{2}\right)<f(u$ ) for $u \in\left(0, \frac{1}{2}\right)$ (so $\left.c^{\prime \prime}<c^{\prime}\right)$. In that case it is easy to show that for typical solutions, the transition $0 \rightarrow \frac{1}{2}$ propagates with speed $c^{\prime}$ while the transition $\frac{1}{2} \rightarrow 1$ propagates with the slower speed $c^{\prime \prime}$, creating a linearly-in- $t$ growing "terrace" on which $u(t, \cdot) \sim \frac{1}{2}$. This and more general such situations were recently studied in [9]. Of course, such reactions are in some sense degenerate, being made of two or more bistable (or other type) reactions "glued" end-to-end. They thus do not resolve the abovementioned question of whether transition fronts must always exists for general "non-degenerate" (i.e., pure) bistable reactions in one dimension. (For pure ignition reactions this can be answered in the affirmative using the general ignition reactions result from [34] - see Theorem 1.2(ii) below. For pure monostable reactions the answer is negative [21.)

In the present paper we prove that existence and uniqueness of transition fronts holds for general inhomogeneous mixed bistable-ignition reactions which satisfy a simple quantitative hypothesis, and that in this case exponentially decaying front-like and spark-like solutions again converge to these fronts as $t \rightarrow \infty$. The same result holds for all pure ignition reactions, without the extra hypothesis. On the other hand, we also show that this hypothesis is not only technical. In fact, we construct an example of a spatially periodic pure bistable reaction for which no transition fronts exist, thus resolving the above question of their existence for pure bistable reactions in the negative. (We note that the latter holds only in the sense of (1.5), for fronts connecting 0 and 1 . Fronts connecting other equilibrium solutions $0 \leq u^{-}<u^{+} \leq 1$ of (1.1) may still exist, such as a front connecting 0 and $\frac{1}{2}$ and another connecting $\frac{1}{2}$ and 1 in the example from the previous paragraph.) This example is, to the best of our knowledge, the first of a non-degenerate (in the sense from the previous paragraph) periodic reaction of any kind in one dimension for which no transition fronts exist, since the monostable reaction examples from [21] are not periodic. (We note that non-existence of traveling fronts was previously proved for bistable reactions on some cylinders with dumbbell-shaped cross-sections and an additional shear flow [4]. The reaction is homogeneous in these and the result hinges instead on the special cross-section and on the added flow. It seems that transition fronts connecting 0 and 1 should not exist in these examples either, although those connecting other equilibria of the PDE in question again may exist.)

Here is our first main result, containing the above claims for (1.1).

Theorem 1.2. Let $f$ be a BI reaction from $(H)$, with $c_{0}$ the unique front speed for $f_{0}$.

(i) Assume that $f_{1}(u)<\frac{c_{0}^{2}}{4} u$ for all $u \in\left(0, \theta_{1}^{\prime}\right]$, with $\theta_{1}^{\prime} \in\left[\theta_{0}, 1\right)$ given by $\int_{\theta_{1}}^{\theta_{1}^{\prime}} f_{0}(u) d u=0$. Then there exists a unique (up to translation in $t$ ) right-moving transition front $w$ for (1.1) (and a unique left-moving one $\tilde{w})$, which then also satisfies $w_{t}>0$. Moreover, solutions with exponentially decaying initial data converge to time shifts of $w, \tilde{w}$ (see Definition 1.3 below). 
(ii) The claims in (i) hold (without the hypothesis on $f_{1}$ ) if $f$ is a pure ignition reaction.

(iii) There exists an $x$-periodic pure bistable reaction $f$ such that there is no (right-or left-moving) transition front for (1.1) in the sense of (1.5). In fact, there are $\varepsilon_{0}, m>0$ such that any solution $0 \leq u \leq 1$ with $u(0, \cdot) \geq \frac{1}{2} \chi_{[-m, m]}$ and $\lim _{x \rightarrow \infty} u(0, x)=0$ takes values within $\left[\varepsilon_{0}, 1-\varepsilon_{0}\right]$ on spatial intervals whose lengths grow linearly in time as $t \rightarrow \infty$.

Remarks. 1. The proof of (i) shows that its existence part extends to mixed bistableignition-monostable reactions with $f_{1}$ satisfying the hypothesis from (i).

2. Note that the hypothesis of (i) is automatically satisfied when $\theta_{1}=\theta_{0}$ (as in [19,20, 22]), or when $\theta_{1}$ is close enough to $\theta_{0}$ (e.g., when $\int_{\theta_{1}}^{4 K\left(4 K-c_{0}^{2}\right)^{-1} \theta_{1}} f_{0}(u) d u>0$ ).

3. In (i), the limits in (1.5) are uniform in $f$ for any fixed $f_{0}, f_{1}, K$, while those in (1.6) and (1.7) below are uniform in $f, u$ for any fixed $f_{0}, f_{1}, K, \theta, Y, \mu, \beta$ (with $l_{f_{0}, \beta}$ in Definition 1.3(b) only depending on $f_{0}, \beta$ ). In (ii) this is true if we also fix $\gamma$ from Definition 1.1.

4. The first claim in (iii) thus proves the conjecture from [7] about existence of such reactions. The second claim describes the reason behind non-existence of transition fronts for these reactions.

Definition 1.3. Let $w, \tilde{w}$ be some right- and left- moving transition fronts for (1.1). We say that solutions with exponentially decaying initial data converge to time shifts of $w, \tilde{w}$ if the following hold for any $Y, \mu>0, \beta>\theta_{0}$, and $a \in \mathbb{R}$.

(a) If $u$ is a (front-like) solution of (1.1) with

$$
\beta \chi_{(-\infty, a]}(x) \leq u(0, x) \leq e^{-\mu(x-a-Y)},
$$

then there is $\tau_{u}$ such that

$$
\lim _{t \rightarrow \infty}\left\|u(t, \cdot)-w\left(t+\tau_{u}, \cdot\right)\right\|_{L^{\infty}}=0
$$

(and similarly for $\tilde{w}$ and $u$ exponentially decaying as $x \rightarrow-\infty$ ).

(b) There is $l_{f_{0}, \beta}<\infty$ such that if $L \geq l_{f_{0}, \beta}$ and $u$ is a (spark-like) solution of (1.1) with

$$
\beta \chi_{[a-L, a+L]}(x) \leq u(0, x) \leq \min \left\{e^{-\mu(x-a-L-Y)}, e^{\mu(x-a+L+Y)}\right\},
$$

then there are $\tau_{u}, \tilde{\tau}_{u}$ such that

$$
\lim _{t \rightarrow \infty}\left\|u(t, \cdot)-w\left(t+\tau_{u}, \cdot\right)-\tilde{w}\left(t+\tilde{\tau}_{u}, \cdot\right)+1\right\|_{L^{\infty}}=0
$$

Let us now turn to the time-inhomogeneous reactions case (1.2). Here we replace in $(\mathrm{H})$ and in Definition 1.1 each $x$ by $t$, while Definition 1.3 refers to convergence to space shifts of $w, \tilde{w}$ and has $w\left(t+\tau_{u}, \cdot\right)$ and $\tilde{w}\left(t+\tilde{\tau}_{u}, \cdot\right)$ replaced by $w\left(t, \cdot+x_{u}\right)$ and $\tilde{w}\left(t, \cdot+\tilde{x}_{u}\right)$. The definition of transition fronts is unchanged.

The time-periodic bistable reaction case was first studied in [1] (the abstract framework of [10] also applies to this case), where it was proved that a unique pulsating front (now satisfying $u(t+p, x)=u(t, x-p c)$ with $p$ the temporal period of $f$ and $c$ the front speed) exists provided the ODE $v^{\prime}=f(t, v)$ has a unique periodic solution $v: \mathbb{R} \rightarrow(0,1)$, which is also unstable.

This was extended to almost-periodic and general stationary ergodic bistable reactions in [23, 24, 26], provided that there is again a single solution $v: \mathbb{R} \rightarrow(0,1)$ of the ODE $v^{\prime}=g(t, v)$ (which must also be unstable) for each $g$ in the $L_{\text {loc }}^{\infty}$-closure of the family of 
all time-translates of $f$. Finally, some general results about transition fronts in stationary ergodic media were proved in [25], which were then applied to show existence of a transition front for $f(t, u)=u(1-u)(u-a(t))$, with $a(t) \in\left[\frac{3}{8}, \frac{5}{8}\right]$ a stationary ergodic process.

The study of time-inhomogeneous ignition reactions is only very recent, with [27,28] proving existence, uniqueness, and stability of transition fronts for ignition reactions with a constant $\tilde{\theta}_{t}\left(\right.$ so $\left.\theta_{1}=\theta_{0}\right)$, also satisfying some additional technical hypotheses.

We now state our second main result, the time-inhomogeneous version of Theorem 1.2 , whose part (ii) also extends [27,28] to general pure ignition reactions.

Theorem 1.4. Let $f$ be a BI reaction from (H) with each $x$ replaced by $t$, with $c_{0}$ the unique front speed for $f_{0}$.

(i) Assume that $f_{1}(u)<\frac{c_{0}^{2}}{4} u$ for all $u \in\left(0, \theta_{0}\right]$. Then the claims in Theorem 1.2( $i$ ) hold for (1.2), with uniqueness of the front up to translation in $x$ and with $w_{x}<0$ instead of $w_{t}>0$.

(ii) The claim in (i) holds (without the hypothesis on $f_{1}$ ) if $f$ is a pure ignition reaction.

(iii) Theorem 1.2(iii) holds for (1.2), with $f$ being a t-periodic pure bistable reaction.

Remark. The remarks after Theorem 1.2 are also valid here.

We close this introduction with an application of our results to the cases of periodic and stationary ergodic reactions.

Corollary 1.5. The following hold under the hypotheses of one of Theorem 1.2(i), Theorem 1.2(ii), Theorem 1.4(i), Theorem 1.4(ii).

(i) If $f$ is spatially/temporally periodic, then the unique transition front is a pulsating front.

(ii) If $f$ is stationary ergodic with respect to spatial/temporal translations (see Section 8 for the precise definition of this), then the unique transition front almost surely has a deterministic asymptotic speed $c>0$ in the sense of $\lim _{|t| \rightarrow \infty} \frac{x_{t}}{t}=c$.

The author thanks Peter Poláčik for a helpful discussion about Theorem 1.2(iii). He also acknowledges partial support by NSF grant DMS-1056327.

\section{Proof of Theorem 1.2(I)}

This follows the lines of a similar proof for ignition reactions in [34]. That proof was done for the PDE

$$
u_{t}=\nabla \cdot(A(x) \nabla u)+q(x) \cdot \nabla u+f(x, u)
$$

with $x \in \mathbb{R} \times \mathbb{T}^{N-1}$, a uniformly elliptic periodic $n \times n$ matrix $A$ and a divergence-free periodic vector field $q$, but not necessarily periodic $f$. We will not consider this setting here.

The existence part of the proof will be done in detail, since it has non-trivial differences from [34, Section 2]. Once this is obtained, proofs of uniqueness of the transition front and of convergence of typical solutions to its time-shifts are virtually identical to those in [34, Sections 3 and 4]. (The ignition property is used in them several times, but it is immediately obvious that $f(x, \cdot)$ being non-increasing on $[0, \theta]$ for each $x \in \mathbb{R}$ suffices instead.) We will therefore only sketch these two parts of the proof here, both for the convenience of the reader as well as for later reference in the proof of Theorem 1.4)(i). 


\section{Existence of a front}

Pick any $\left(f_{0}\right.$-dependent $) \varepsilon_{0} \in\left(0, \theta_{0}\right)$ such that $\int_{0}^{1-\varepsilon_{0}} f_{0}(u) d u>0$ and $1-\varepsilon_{0}$ is greater than any point of maximum of $\frac{f_{0}(u)}{u}$. Using (1.4), it is easy to construct $v: \mathbb{R} \rightarrow[0,1]$ satisfying $v^{\prime \prime}+f_{0}(v) \geq 0$, supported on $\mathbb{R}^{-}$, and equal to $1-\varepsilon_{0}$ for $x \ll-1$. One can take $v \equiv 1-\varepsilon_{0}$ on $(-\infty, 0]$, let $v^{\prime \prime}+f_{0}(v)=0$ (with $v(0)=1-\varepsilon_{0}$ and $\left.v^{\prime}(0)=0\right)$ on $(0, r)$, where $r>0$ is smallest such that $v(r)=0$, and let $v \equiv 0$ on $[r, \infty)$ (then we shift $v$ by $r$ to the left). The existence of $r$ follows from multiplying $v^{\prime \prime}+f_{0}(v)=0$ by $v^{\prime}$ and integrating over $(0, x)$, which yields $\frac{1}{2} v^{\prime}(x)^{2}=F_{0}\left(1-\varepsilon_{0}\right)-F_{0}(v(x))$, with

$$
F_{0}(u):=\int_{0}^{u} f_{0}(s) d s .
$$

Since $F_{0}(u)<F_{0}\left(1-\varepsilon_{0}\right)$ for $u \in\left[0,1-\varepsilon_{0}\right)$ due to $F_{0}\left(1-\varepsilon_{0}\right)=\int_{0}^{1-\varepsilon_{0}} f_{0}(u) d u>0$, we see that $v^{\prime}$ cannot change sign before $v$ hits 0 . So $v^{\prime}$ stays negative, and then $v$ must hit 0 at some finite $r$ because $F_{0}(u)<F_{0}\left(1-\varepsilon_{0}\right)$ for $u \in\left[0,1-\varepsilon_{0}\right)$.

We now let $u_{n}$ be the solution of (1.1) with initial condition $u_{n}(0, x)=v(x+n)$. Then $f \geq f_{0}$ and $1-\varepsilon_{0}>\theta_{0}$, together with well known spreading results [2,11], imply that $\lim _{t \rightarrow \infty} u_{n}(t, x)=1$ locally uniformly. Hence there is (minimal) $\tau_{n}$ such that $u_{n}\left(\tau_{n}, 0\right)=\frac{1}{2}$, and then finite speed of propagation (e.g., $u_{n}(t, x) \leq e^{-\sqrt{\xi}(x+n-2 \sqrt{\xi} t)}$ for $\xi:=\max _{u \in(0,1]} \frac{f_{1}(u)}{u}$, since the exponential is a super-solution of (1.1) when we define $f(x, u)=0$ for $u>1$ ) easily shows $\tau_{n} \rightarrow \infty$. We let $\tilde{u}_{n}(t, x):=u_{n}\left(t+\tau_{n}, x\right)$, so that $\tilde{u}_{n}$ solves (1.1) on $\left(-\tau_{n}, \infty\right) \times \mathbb{R}$, with $\tilde{u}_{n}(0,0)=\frac{1}{2}$. Parabolic regularity now shows that some subsequence of $\tilde{u}_{n}$ converges in $C_{\text {loc }}^{1,2}$ to an entire solution $w$ of (1.1) with $w(0,0)=\frac{1}{2}$. We also have $w_{t} \geq 0$ due to $\left(u_{n}\right)_{t} \geq 0$, which follows from $\left(u_{n}\right)_{t}(0, \cdot) \geq 0$ and the maximum principle for $\left(u_{n}\right)_{t}$. To show that $w$ is indeed a transition front, we now only need to prove that the limits (1.5) hold uniformly in $t \in \mathbb{R}$. (This and $w(0,0)=\frac{1}{2}$ also imply $w_{t} \not \equiv 0$, and the strong maximum principle for $w_{t}$ then proves $w_{t}>0$ as well.) This will in turn be proved by showing that

$$
\sup _{n \in \mathbb{N} \& t \geq T_{\varepsilon}} \operatorname{diam}\left\{x \in \mathbb{R} \mid u_{n}(t, x) \in[\varepsilon, 1-\varepsilon]\right\}<\infty
$$

for each $\varepsilon>0$ and some $n$-independent $T_{\varepsilon}<\infty$.

We now pick $\zeta<\frac{c_{0}^{2}}{4}$ and $\theta_{1}^{\prime \prime}>\theta_{1}^{\prime}$ (both depending only on $f_{0}, f_{1}$ ) so that

$$
f_{1}(u)<\zeta u \quad \text { for } u \in\left(0, \theta_{1}^{\prime \prime}\right],
$$

and let $c_{\zeta}:=2 \sqrt{\zeta}$ and $c_{\xi}:=(\xi+\zeta) \zeta^{-1 / 2}$. It is well known that $c_{0} \leq c_{1} \leq 2 \sqrt{\xi}$ (with $c_{1}$ the unique front speed for $f_{1}$ ), hence we have $\zeta<\xi$ and $c_{\zeta}<c_{0} \leq c_{\xi}$.

Finally, for each $n \in \mathbb{N}$ and $t \geq 0$ we let

$$
\begin{gathered}
X_{n}(t):=\max \left\{x \in \mathbb{R} \mid u_{n}(t, x) \geq \theta_{1}^{\prime \prime}\right\}, \\
Y_{n}(t):=\min \left\{y \in \mathbb{R} \mid u_{n}(t, x) \leq e^{-\sqrt{\zeta}(x-y)} \text { for all } x \in \mathbb{R}\right\} .
\end{gathered}
$$

We note that the proof in [34] (see also Section 3) defined $X_{n}(t)$ to be the largest $x$ for which $f(x, u)<\zeta u$ does not hold for all $u \in\left(0, u_{n}(t, x)\right)$ (which is then smaller than our $X_{n}(t)$ ), but our definition will suffice here. Also note that $X_{n}$ and $Y_{n}$ are both non-decreasing because $\left(u_{n}\right)_{t} \geq 0$, and we have $X_{n}(0)=X_{0}(0)-n$ and $Y_{n}(0)=Y_{0}(0)-n$. Since $\theta_{1}^{\prime \prime}$ is smaller than 
any point of maximum of $\frac{f_{0}(u)}{u}$ (due to $\zeta<\max _{u \in(0,1]} \frac{f_{0}(u)}{u}$, which follows from $\zeta<\frac{c_{0}^{2}}{4}$ ) we obtain $\theta_{1}^{\prime \prime}<1-\varepsilon_{0}$. Hence $X_{n}(t)$ is finite, while $Y_{n}(t)$ is finite by the following crucial lemma.

Lemma 2.1. (i) For any $n$ and $t \geq t^{\prime} \geq 0$ we have

$$
Y_{n}(t)-Y_{n}\left(t^{\prime}\right) \leq c_{\xi}\left(t-t^{\prime}\right) .
$$

If also $X_{n}(t) \leq Y_{n}\left(t^{\prime}\right)$, then in fact

$$
Y_{n}(t)-Y_{n}\left(t^{\prime}\right) \leq c_{\zeta}\left(t-t^{\prime}\right)
$$

(ii) For every $\varepsilon>0$ there is $r_{\varepsilon}<\infty$ such that for any $n$ and $t \geq t^{\prime} \geq 0$ we have

$$
\inf _{\left|x-X_{n}\left(t^{\prime}\right)\right| \leq c_{0}\left(t-t^{\prime}\right)-r_{\varepsilon}} u_{n}(t, x) \geq 1-\varepsilon .
$$

This $r_{\varepsilon}$ only depends on $\varepsilon, f_{0}, f_{1}, K$.

Proof. (i) The first claim follows from $e^{-\sqrt{\zeta}\left(x-Y_{n}\left(t^{\prime}\right)-c_{\xi}\left(t-t^{\prime}\right)\right)}$ being a super-solution of (1.1). The second claim follows from $w(t, x):=e^{-\sqrt{\zeta}\left(x-Y_{n}\left(t^{\prime}\right)-c_{\zeta}\left(t-t^{\prime}\right)\right)}$ satisfying $w_{t}=w_{x x}+\zeta w$, while $u_{n}$ is a sub-solution of this PDE on $\left(t^{\prime}, t\right) \times\left(X_{n}(t), \infty\right)$ due to (2.3), the definition of $X_{n}$, and due to $X_{n}$ being non-decreasing (note that $w \geq 1>u_{n}$ on $\left(t^{\prime}, t\right) \times\left(-\infty, X_{n}(t)\right]$ because $\left.X_{n}(t) \leq Y_{n}\left(t^{\prime}\right)\right)$.

(ii) Note that (2.6) will follow from $f(x, u) \geq f_{0}(u)$ and well-known spreading results (i.e., spreading with speed $c_{0}$ for $u_{t}=u_{x x}+f_{0}(u)$ [2,11]) once we show for each $L<\infty$ existence of $T<\infty$ (depending on $\left.L, \theta_{1}^{\prime \prime}, f_{0}, f_{1}, K\right)$ such that under the hypotheses of (ii) we have

$$
\inf _{\left|x-X_{n}\left(t^{\prime}\right)\right| \leq L} u_{n}\left(t^{\prime}+T, x\right) \geq \theta_{1}^{\prime \prime} .
$$

(Here $\theta_{1}^{\prime \prime}$ can be replaced by any constant larger than $\theta_{1}$. Moreover, an $L$ such that (2.7) indeed implies (2.6) only depends on $\theta_{1}^{\prime \prime}, f_{0}$, while $\theta_{1}^{\prime \prime}$ only depends on $f_{0}, f_{1}$. Hence $r_{\varepsilon}$ will only depend on $\varepsilon, f_{0}, f_{1}, K$.) We will now prove (2.7) for any fixed $L$.

First we claim that $u_{n}\left(t^{\prime}, x\right) \geq \theta_{1}$ for $x \leq X_{n}\left(t^{\prime}\right)$. This is because $f\left(x, \theta_{1}\right) \leq 0$, so the set $I_{n}(t):=\left\{x \mid u_{n}(t, x) \geq \theta_{1}\right\}$ cannot acquire new connected components due to the maximum principle, and because $\left(u_{n}\right)_{t} \geq 0$, so $I_{n}(t)$ cannot split into several connected components either. Since $I_{n}(0)$ is some interval $(-\infty, \iota-n]$, it follows that $I_{n}(t)$ is some interval $\left(-\infty, \iota_{n}(t)\right]$.

Assume now that (2.7) does not hold for some $L$. Then for each $k \in \mathbb{N}$ we can find $n_{k}$ and $\left(t_{k}^{\prime}, x_{k}\right) \in[0, \infty) \times[-L, L]$ such that $u_{n_{k}}\left(t_{k}^{\prime}+k, X_{n_{k}}\left(t_{k}^{\prime}\right)+x_{k}\right)<\theta_{1}^{\prime \prime}$. Then each $w_{k}(t, x):=u_{n_{k}}\left(t+t_{k}^{\prime}, x+X_{n_{k}}\left(t_{k}^{\prime}\right)\right)$ satisfies (1.1) on $\mathbb{R}^{+} \times \mathbb{R}$, with $f$ replaced by $g_{k}(x, u):=$ $f\left(x+X_{n_{k}}\left(t_{k}^{\prime}\right), u\right)$. Parabolic regularity, $f_{0} \leq f \leq f_{1}$, and $f$ being $K$-Lipschitz show that a subsequence of $w_{k}$ converges in $C_{\text {loc }}^{1,2}\left(\mathbb{R}^{+} \times \mathbb{R}\right)$ to some solution $\tilde{w}$ of (1.1), with $f$ replaced by some $K$-Lipschitz $g$ such that $f_{0} \leq g \leq f_{1}$. Moreover, $\tilde{w}_{t}>0, \tilde{w}(0, \cdot) \geq \theta_{1} \chi_{\mathbb{R}^{-}}, \tilde{w}(0,0) \geq \theta_{1}^{\prime \prime}$, and $w(x):=\lim _{t \rightarrow \infty} \tilde{w}(t, x)$ solves $w^{\prime \prime}+g(x, w)=0$ on $\mathbb{R}$ and satisfies $w\left(x_{0}\right) \leq \theta_{1}^{\prime \prime}$ for some $\left|x_{0}\right| \leq L$. Since $w \leq 1$, it also follows that $w<1$.

We thus obtain $w^{\prime \prime}+f_{0}(w) \leq 0$ and $\theta_{1} \chi_{\mathbb{R}^{-}} \leq w<1$ as well as $w(0) \in\left[\theta_{1}^{\prime \prime}, 1\right)$. Multiplying the former by $w^{\prime}$ and integrating over $(a, 0)$, with $a \in[-\infty, 0)$ smallest such that $w^{\prime}$ does not change sign on $(a, 0)$ (hence $w^{\prime}(a)=0$ ) yields

$$
\operatorname{sgn}(w(0)-w(a))\left[\frac{w^{\prime}(0)^{2}}{2}+F_{0}(w(0))-F_{0}(w(a))\right] \leq 0 .
$$


From $w(0) \geq \theta_{1}^{\prime \prime}, w(a) \geq \theta_{1}$, and $\int_{\theta_{1}}^{\theta_{1}^{\prime \prime}} f_{0}(u) d u>0$ we obtain

$$
\operatorname{sgn}\left(F_{0}(w(0))-F_{0}(w(a))\right)=\operatorname{sgn}(w(0)-w(a)),
$$

so we must have $w(a) \geq w(0)$.

If $a>-\infty$, we let $a^{\prime} \in[-\infty, a)$ be smallest such that $w^{\prime}$ does not change sign on $\left(a^{\prime}, a\right)$, and the same argument yields

$$
\operatorname{sgn}\left(w(a)-w\left(a^{\prime}\right)\right)\left[F_{0}(w(a))-F_{0}\left(w\left(a^{\prime}\right)\right)\right] \leq 0 .
$$

Since $w(a) \in\left[\theta_{1}^{\prime \prime}, 1\right), w\left(a^{\prime}\right) \in\left[\theta_{1}, 1\right]$, and $\int_{\theta_{1}}^{\theta_{1}^{\prime \prime}} f_{0}(u) d u>0$, we see that this is only possible if $w\left(a^{\prime}\right)=w(a)$. But then $a^{\prime}=-\infty$ and $w \equiv w(a)$ on $(-\infty, a)$, a contradiction with $w^{\prime \prime}+f_{0}(w) \leq 0$ because $f_{0}>0$ on $\left[\theta_{1}^{\prime \prime}, 1\right)$.

If now $a=-\infty$, we must have $f_{0}(w(-\infty)) \leq 0$ (and $w(-\infty) \geq \theta_{1}^{\prime \prime}$ ) which leaves us with $w(-\infty)=1$. Running the above argument on $(-\infty, b)$, with $b \in[0, \infty]$ largest such that $w^{\prime}$ does not change sign on $(-\infty, b)$, then yields

$$
\operatorname{sgn}(w(b)-1)\left[F_{0}(w(b))-F_{0}(1)\right] \leq 0 \text {. }
$$

The properties of $f_{0}$ now force $w(b)=1$. Hence $b=\infty$ and $w \equiv 1$, a contradiction with $w\left(x_{0}\right) \leq \theta_{1}^{\prime \prime}$.

This proves (2.7). Notice that the $T$ we obtained is independent of $f$ because the contradiction argument can be run uniformly in all $f$ from $(\mathrm{H})$ (we pick $\left\{\left(f_{k}, n_{k}, t_{k}^{\prime}, x_{k}\right)\right\}_{k=1}^{\infty}$ instead of $\left.\left\{\left(n_{k}, t_{k}^{\prime}, x_{k}\right)\right\}_{k=1}^{\infty}\right)$. Thus $T=T\left(L, \theta_{1}^{\prime \prime}, f_{0}, f_{1}, K\right)$ and as mentioned above, it follows that $r_{\varepsilon}$ depends only on $\varepsilon, f_{0}, f_{1}, K$.

Having proven the lemma, we now easily obtain

$$
\sup _{n \in \mathbb{N} \& t \geq 0}\left|Y_{n}(t)-X_{n}(t)\right| \leq C
$$

for some $C=C\left(f_{0}, f_{1}, K\right)$. The uniform bound $X_{n}(t)-Y_{n}(t) \leq C\left(f_{0}, f_{1}\right)$ is obvious from the definition of $X_{n}, Y_{n}$ (since $\varepsilon_{0}, \theta_{1}^{\prime \prime}, \zeta$ only depend on $f_{0}, f_{1}$ ), so we are left with proving $Y_{n}(t)-X_{n}(t) \leq C\left(f_{0}, f_{1}, K\right)$.

Note that the claims of Lemma 2.1(i) together prove

$$
Y_{n}(t)-Y_{n}\left(t^{\prime}\right) \leq c_{\zeta}\left(t-t^{\prime}\right) \quad \text { when } Y_{n}(t)-X_{n}(t) \geq c_{\xi}\left(t-t^{\prime}\right),
$$

and Lemma 2.1(ii) shows

$$
X_{n}(t)-X_{n}\left(t^{\prime}\right) \geq c_{0}\left(t-t^{\prime}\right)-r_{\varepsilon_{0}}
$$

(recall that we have $1-\varepsilon_{0} \geq \theta_{1}^{\prime \prime}$ ). Let $S:=\left|Y_{n}(0)-X_{n}(0)\right|$ (which is independent of $n, f$ ) and $C:=S+c_{\xi} r_{\varepsilon_{0}}\left(c_{0}-c_{\zeta}\right)^{-1}$. If $t \geq 0$ is the first time such that $Y_{n}(t)-X_{n}(t)=C$ (note that $Y_{n}(t)-X_{n}(t)$ is lower semi-continuous because so is $X_{n}$, and $Y_{n}$ is continuous), then $t \geq r_{\varepsilon_{0}}\left(c_{0}-c_{\zeta}\right)^{-1}$ by Lemma 2.1(i) and we let $t^{\prime}:=t-r_{\varepsilon_{0}}\left(c_{0}-c_{\zeta}\right)^{-1}$. But now (2.9) and (2.10) yield $X_{n}(t)-X_{n}\left(t^{\prime}\right) \geq Y_{n}(t)-Y_{n}\left(t^{\prime}\right)$, a contradiction with the choice of $t$. This proves (2.8).

Finally, let us define

$$
\begin{aligned}
& Z_{n, \varepsilon}^{-}(t):=\max \left\{y \in \mathbb{R} \mid u_{n}(t, x)>1-\varepsilon \text { for all } x<y\right\} \\
& Z_{n, \varepsilon}^{+}(t):=\min \left\{y \in \mathbb{R} \mid u_{n}(t, x)<\varepsilon \text { for all } x>y\right\}
\end{aligned}
$$


Continuity of $Y_{n}$ and (2.8) show that the non-decreasing function $X_{n}$ can have jumps no longer than $2 C$. This, $\left(u_{n}\right)_{t} \geq 0$, and Lemma 2.1(ii) (together with $X_{n}(0) \leq-n$ and $u_{n}(0, x) \geq 1-\varepsilon_{0}>\theta_{1}^{\prime \prime}$ for $x \leq-n-r$; see the construction of the initial data $v$ above), imply that there is $T_{\varepsilon}$ such that $Z_{n, \varepsilon}^{-}\left(t+T_{\varepsilon}\right) \geq X_{n}(t)$ for any $t \geq 0$, and $T_{\varepsilon}$ depends only on $\varepsilon, f_{0}, f_{1}, K$. From the definition of $Y_{n}$ and Lemma 2.1(i) we also have

$$
Z_{n, \varepsilon}^{+}\left(t+T_{\varepsilon}\right) \leq Y_{n}\left(t+T_{\varepsilon}\right)+\zeta^{-1 / 2}|\log \varepsilon| \leq Y_{n}(t)+c_{\xi} T_{\varepsilon}+\zeta^{-1 / 2}|\log \varepsilon|
$$

so (2.8) allows us to conclude for each $t \geq 0$,

$$
Z_{n, \varepsilon}^{+}\left(t+T_{\varepsilon}\right)-Z_{n, \varepsilon}^{-}\left(t+T_{\varepsilon}\right) \leq c_{\xi} T_{\varepsilon}+\zeta^{-1 / 2}|\log \varepsilon|+C \quad\left(=: L_{\varepsilon}\right) .
$$

But this is precisely (2.2), and the proof is finished.

Notice that this also shows that the upper bound $L_{\varepsilon}$ on the left-hand side of (2.2) only depends on $\varepsilon, f_{0}, f_{1}, K$, so as claimed in Remark 3 after Theorem 1.2, the limits in (1.5) are indeed uniform in all $f$ satisfying $(\mathrm{H})$ with some fixed $f_{0}, f_{1}, K$.

Notice also that so far we used neither $\theta>0$ nor $\theta_{1}>0$. Hence existence of fronts extends to mixed bistable-ignition-monostable reactions.

\section{Uniqueness of the front and convergence of typical solutions to it}

As mentioned above, these proofs are essentially identical to their analogs in [34, Sections 3 and 4]. We only sketch them and refer the reader to [34] for any details skipped here.

Replace $\varepsilon_{0}$ from the existence proof by the minimum of itself and $\frac{\theta}{2}$ (hence it now depends on $\left.f_{0}, \theta\right)$. Then let $v$ and $u:=u_{0}$ be from the existence proof (i.e., $u$ solves (1.1) with $u(0, \cdot)=v)$, and let

$$
\begin{aligned}
X_{u}(t) & :=\max \left\{x \in \mathbb{R} \mid u(t, x) \geq \theta_{1}^{\prime \prime}\right\}, \\
Y_{u}(t) & :=\min \left\{y \in \mathbb{R} \mid u(t, x) \leq e^{-\sqrt{\zeta}(x-y)} \text { for all } x \in \mathbb{R}\right\}, \\
Z_{u, \varepsilon}^{-}(t) & :=\max \{y \in \mathbb{R} \mid u(t, x)>1-\varepsilon \text { for all } x<y\}, \\
Z_{u, \varepsilon}^{+}(t) & :=\min \{y \in \mathbb{R} \mid u(t, x)<\varepsilon \text { for all } x>y\}
\end{aligned}
$$

for $t \geq 0$. We also define

$$
Z_{u}(t):=Z_{u, \varepsilon_{0}}^{-}(t)
$$

and note that (2.8), Lemma 2.1, and $Z_{n, \varepsilon}^{-}\left(t+T_{\varepsilon}\right) \geq X_{n}(t)$ proved above show

$$
\sup _{t \geq T_{\varepsilon_{0}}}\left|Y_{u}(t)-Z_{u}(t)\right| \leq C_{2}
$$

with $C_{2}=C_{2}\left(f_{0}, f_{1}, K, \theta\right)$ and $T_{\varepsilon}=T_{\varepsilon}\left(f_{0}, f_{1}, K\right)$.

Let now $0 \leq w \leq 1$ be any transition front for (1.1), define $X_{w}(t), Y_{w}(t), Z_{w, \varepsilon}^{-}(t), Z_{w, \varepsilon}^{+}(t), Z_{w}(t)$ as above but with $w$ in place of $u$ and for any $t \in \mathbb{R}$ (here $Y_{w}(t)$ might, in principle, be $\infty$ ). Also define

$$
L_{w}:=\sup _{t \in \mathbb{R}}\left\{Z_{w, \varepsilon_{0}}^{+}(t)-Z_{w, \varepsilon_{0}}^{-}(t)\right\}
$$

which is finite because $w$ is a transition front.

First, [34, Lemma 3.1] shows

$$
\sup _{t \in \mathbb{R}}\left|Y_{w}(t)-Z_{w}(t)\right| \leq \tilde{C}_{2}
$$


with $\tilde{C}_{2}$ depending on $f_{0}, f_{1}, K, \theta$ (and also on $L_{w}$ if $w_{t} \ngtr 0$ ). Consider first the case $w_{t}>0$. Then (2.13) is obtained by letting for $h>0$,

$$
Y_{w, h}(t):=\min \left\{y \in \mathbb{R} \mid w(t, x) \leq h+e^{-\sqrt{\zeta}(x-y)} \text { for all } x \in \mathbb{R}\right\}<\infty,
$$

and proving for all small $h>0$,

$$
\sup _{t \in \mathbb{R}}\left|Y_{w, h}(t)-X_{w}(t)\right| \leq C_{2}\left(f_{0}, f_{1}, K\right)
$$

(then we take $h \rightarrow 0$, and afterwards conclude (2.13) as we did (2.12)). Finally, (2.14) is obtained as in the existence proof, using Lemma 2.1 for $X_{w}, Y_{w, h}$ and any $t \geq t^{\prime}$, which holds for any $h>0$ such that

$$
f_{1}(u)<\zeta(u-h) \quad \text { for } u \in\left(h, \theta_{1}^{\prime \prime}\right] .
$$

This is true for all small enough $h>0$ due to (2.3).

If now $w_{t} \ngtr 0$ and $h>0$ is small enough, then Lemma 2.1(i) holds for $X_{w}, Y_{w, h}$ and $t \geq t^{\prime}$, but with $X_{w}(t) \leq Y_{w, h}\left(t^{\prime}\right)$ replaced by $\sup _{t^{\prime} \leq s \leq t} X_{w}(s) \leq Y_{w, h}\left(t^{\prime}\right)$. Also, Lemma 2.1(ii) easily holds with (2.6) replaced by

$$
\inf _{x \leq X_{w}\left(t^{\prime}\right)-L_{w}+c_{0}\left(t-t^{\prime}\right)-r_{\varepsilon}} w(t, x) \geq 1-\varepsilon .
$$

This is because $\varepsilon_{0}<\theta_{0} \leq \theta_{1}^{\prime \prime}<1-\varepsilon_{0}$, so (2.7) can be replaced in the proof by the obvious

$$
\inf _{x \leq X_{w}\left(t^{\prime}\right)-L_{w}} w\left(t^{\prime}, x\right) \geq \theta_{1}^{\prime \prime}
$$

This version of Lemma 2.1 yields (2.14), with $C_{2}$ also depending on $L_{w}$.

Next, [34, Lemma 3.2] shows that for each $\varepsilon>0$ there is $\delta>0$, depending also on $f_{0}, f_{1}, K, \theta$ (and also on $L_{w}$ if $w_{t} \ngtr 0$ ), such that the following holds for any $t_{0} \geq 1, t_{1} \in \mathbb{R}$, and $t \geq t_{0}$ :

$$
\text { if } \pm\left[w\left(t_{1}, \cdot\right)-u\left(t_{0}, \cdot\right)\right] \leq \delta, \text { then } \pm\left[w\left(t+t_{1}-t_{0}, \cdot\right)-u(t, \cdot)\right] \leq \varepsilon
$$

Of course, $u, w$ are the particular solutions of (1.1) considered here (in particular, one needs to use that they decay exponentially as $x \rightarrow \infty$ ). The proof of the + case (without loss assume $t_{1}=t_{0}$, otherwise shift $w$ in time) is via the construction of a super-solution of (1.1) of the form

$$
z_{+}(t, x):=u\left(t+\frac{\varepsilon}{\Omega}\left(1-e^{-\sqrt{\zeta}\left(c_{0}-c_{\zeta}\right)\left(t-t_{0}\right) / 4}\right), x\right)+b_{\varepsilon} e^{-\sqrt{\zeta}\left(x-Y_{w}\left(t_{0}\right)-c_{\zeta}\left(t-t_{0}\right)\right) / 2},
$$

with $\Omega$ large so that $\left|u_{t}\right| \leq \Omega$ for $t \geq 1$ (such $\Omega=\Omega(K)$ exists by parabolic regularity) and $b_{\varepsilon}>0$ small and depending also on $f_{0}, f_{1}, K, \theta$ (and also on $L_{w}$ if $w_{t} \ngtr 0$ ). That such $b_{\varepsilon}$ exists follows from $u_{t}>0$, the strong maximum principle for $u_{t}$, and (recall that $\varepsilon_{0} \leq \frac{\theta}{2}$ ) $\sup _{t \geq 1}\left\{Z_{u, \theta / 2}^{+}(t)-Z_{u, \theta / 2}^{-}(t)\right\}<\infty$ - which together show that $u_{t}(t, x)$ is uniformly positive where $u(t, x) \in\left[\frac{\theta}{2}, 1-\frac{\theta}{2}\right]$ — as well as from $f$ being non-increasing in $u$ on $[0, \theta]$ and on $[1-\theta, 1]$ (we also let $f(x, u) \leq 0$ for $u>1$ ). Note that a crucial property of $z_{+}$is that the second term travels with speed $c_{\zeta}$, which is smaller than the lower bound $c_{0}$ on the speed of propagation of the first term. Hence $z_{+}\left(t, \cdot+x_{t}\right)-u\left(t+\frac{\varepsilon}{\Omega}, \cdot+x_{t}\right)$, with $x_{t}:=\max \left\{x \in \mathbb{R} \mid u(t, x)=\frac{1}{2}\right\}$, converges locally uniformly to 0 as $t \rightarrow \infty$. A simple argument 34 then concludes the + case of (2.17) with $\delta$ depending on $b_{\varepsilon}$ (specifically, $\delta=b_{\varepsilon}^{2}$ for that particular choice of $b_{\varepsilon}$, and then one obtains (2.17) with $2 \varepsilon$ instead of $\varepsilon$ ). 
The proof of the - case of (2.17) is similar, using the sub-solution

$$
z_{-}(t, x):=u\left(t-\frac{\varepsilon}{\Omega}\left(1-e^{-\sqrt{\zeta}\left(c_{0}-c_{\zeta}\right)\left(t-t_{0}\right) / 4}\right), x\right)-b_{\varepsilon} e^{-\sqrt{\zeta}\left(x-Y_{u}\left(t_{0}\right)-c_{\zeta}\left(t-t_{0}\right)\right) / 2}
$$

as well as Lemma 2.1(ii) for $w$ (the latter is needed because $\lim _{x \rightarrow-\infty} z_{-}(t, x)=-\infty$ ).

These estimates now easily show (see [34, Lemma 3.3]) that

$$
\tau_{w}:=\inf \left\{\tau \in \mathbb{R} \mid \liminf _{t \rightarrow \infty} \inf _{x \in \mathbb{R}}[w(t+\tau, x)-u(t, x)] \geq 0\right\}
$$

is a finite number, and hence also

$$
\liminf _{t \rightarrow \infty} \inf _{x \in \mathbb{R}}\left[w\left(t+\tau_{w}, x\right)-u(t, x)\right] \geq 0 .
$$

Then it is shown in [34, Lemma 3.4] that in fact

$$
\lim _{t \rightarrow \infty}\left\|w\left(t+\tau_{w}, \cdot\right)-u(t, \cdot)\right\|_{L^{\infty}}=0
$$

Indeed, if this were false, then (2.17), (2.21), (2.12), (2.13), and the strong maximum principle would imply

$$
\liminf _{t \rightarrow \infty} \inf _{\left|x-x_{t}\right| \leq L}\left[w\left(t+\tau_{w}, x\right)-u(t, x)\right]>0
$$

for any $L<\infty$. This, (2.17), the definition of $\tau_{w}$, and $f$ being non-increasing in $u$ on $[0, \theta]$ and on $[1-\theta, 1]$ can be shown to yield a contradiction (also using parabolic regularity).

Hence each transition front must converge in $L^{\infty}$ to some time-shift of $u$ as $t \rightarrow \infty$. Finally, this convergence is shown in [34, Lemma 3.5] to be uniform in all $f$ satisfying $(\mathrm{H})$ (for any fixed $f_{0}, f_{1}, K, \theta$ ) and all $w$ with $L_{w} \leq C$ (for any fixed $C<\infty$ ). Indeed, if this were not true, one could obtain a counter-example to (2.22) by passing to a subsequence of more and more slowly converging couples $u, w$ as above (each with its own $f$; this again uses parabolic regularity and $f$ being $K$-Lipschitz).

Since this uniformity includes any translations of $f$ in $x$, we obtain that if $w_{1}, w_{2}$ are two transition fronts for $f$, the solutions $u_{n}$ of (1.1) with initial conditions $u_{n}(0, x):=v(x+n)$ converge uniformly quickly (in $n$ ) as $t \rightarrow \infty$ to some time translates (by $\tau_{1, n}$ and $\tau_{2, n}$ ) of $w_{1}, w_{2}$. Obviously $\tau_{1, n}, \tau_{2, n} \rightarrow-\infty$ as $n \rightarrow \infty$, which together with the stability result (2.17) shows that for any $t^{\prime} \in \mathbb{R}$ and $\varepsilon>0$, there is $\tau_{t^{\prime}, \varepsilon}$ such that

$$
\sup _{t \geq t^{\prime} \& x \in \mathbb{R}}\left|w_{1}(t, x)-w_{2}\left(t+\tau_{t^{\prime}, \varepsilon}, x\right)\right|<\varepsilon .
$$

Since $t^{\prime} \in \mathbb{R}$ and $\varepsilon>0$ are arbitrary, it follows that $w_{1}(\cdot, \cdot) \equiv w_{2}(\cdot-\tau, \cdot)$ for some $\tau \in \mathbb{R}$. Thus there is a unique transition front (up to translation in $t$ ), which then must be the one constructed in the existence proof. That front satisfies $w_{t}>0$ and has $L_{w}$ uniformly bounded in $f$ (for any fixed $f_{0}, f_{1}, K, \theta$ ), hence we find that, in fact, the constants in the above results do not depend on $L_{w}$.

This proves the uniqueness claim of Theorem 1.2(i). The proof of the convergence claim for front-like solutions is very similar to the uniqueness proof, but with $u$ now being the unique transition front, while $w$ being the front-like solution (so the notation from Definition 1.3 is reversed). There are only two significant differences. The first is that $Y_{w}$ must now be defined with $\sqrt{\zeta}$ replaced by $\mu$ so that it is finite, and $\sqrt{\zeta}$ is replaced by $2 \mu$ in $(2.18)$ (this uses $\mu \leq \frac{1}{2} \sqrt{\zeta}$, which can be assumed without loss). The second is that while now we do not have the uniform limits (1.5), we can instead easily bound $w$ from above by the new 
super-solution (2.18) and from below by the original sub-solution (2.19) (with two different $t_{0}$ ). This, (2.12) (with $t \in \mathbb{R}$ ), and (2.4) (which now holds with $c_{\xi}:=\left(K+\mu^{2}\right) \mu^{-1}$ ) then prove (2.13) (with $t \geq 0$ ), even though the crucial estimate (2.5) does not anymore hold for the new $Y_{w}$ and some $c_{\zeta}<c_{0}$.

The proof for spark-like solutions is identical, but restricted to $x \in \mathbb{R}^{+}$(and then to $x \in \mathbb{R}^{-}$ and the unique left-moving front). Finally, the second claim in Remark 3 after Theorem 1.2 is also proved as in [34] - if it were false, one could use parabolic regularity to construct a reaction satisfying the hypotheses but not the result on convergence of front-like or spark-like solutions to the transition fronts.

\section{Proof of Theorem 1.2(II)}

This is an immediate corollary of [34, Theorem 1.3]. The latter is the same result for (2.1) and ignition reactions (see Definition 1.1) satisfying the following hypothesis (which we state here in the case of (1.1), with $\theta_{0}$ from $(\mathrm{H})$ and $c_{0}$ the unique front speed for $\left.f_{0}\right)$ :

There are $\zeta<\frac{c_{0}^{2}}{4}$ and $\eta>0$ such that

$$
\inf _{x \in \mathbb{R} \& u \in\left[\alpha_{f}(x), \theta_{0}\right]} \sup _{|y-x| \leq \eta^{-1}} f(y, u) \geq \eta, \quad \text { where } \alpha_{f}(x):=\inf \{u \in(0,1) \mid f(x, u) \geq \zeta u\} .
$$

This hypothesis automatically holds for all pure ignition reactions (even without the sup and with $f(y, u)$ replaced by $f(x, u))$, with any $\zeta \in\left(0, \frac{c_{0}^{2}}{4}\right)$ and $\eta$ depending on $\zeta, \theta_{1}, K, \gamma$ (the latter from Definition [1.1). We also note that in the proof of [34, Theorem 1.3], $X_{n}$ is replaced by the smaller

$$
\tilde{X}_{n}(t):=\max \left\{x \in \mathbb{R} \mid u_{n}(t, x) \geq \alpha_{f}(x)\right\},
$$

that the extra hypothesis on $f_{1}$ from Theorem 1.2(i) can be replaced by (3.1) thanks to the fact that any bounded solution to $0=u_{x x}+f(x, u)$ with $f \geq 0$ must be constant, and that the existence part of that result extends to mixed ignition-monostable reactions.

\section{Proof of Theorem 1.2(III)}

We start with a periodic stationary solution $p$ of (1.1) with $f=f_{0}$, where $f_{0}$ is any homogeneous pure bistable reaction. It is well known that such solutions are obtained by solving the $\mathrm{ODE} p^{\prime \prime}+f_{0}(p)=0$ on $\mathbb{R}$, with any $p(0) \in\left[\theta_{0}, \theta_{0}^{\prime}\right)$ and $p^{\prime}(0)=0$, where $\theta_{0}^{\prime} \in\left(\theta_{0}, 1\right)$ is given by $\int_{0}^{\theta_{0}^{\prime}} f_{0}(u) d u=0$. It is easy to show (by multiplying the ODE by $p^{\prime}$ and integrating on any interval where $p^{\prime}$ does not change sign) that $p(\mathbb{R})=[P, p(0)]$, where $\int_{P}^{p(0)} f_{0}(u) d u=0$. We pick $p(0)=\frac{1}{4}\left(\theta_{0}+3 \theta_{0}^{\prime}\right)$ and denote $M$ the period of the corresponding solution $p$. Next we let $m>0$ be such that $p \geq \frac{1}{4}\left(2 \theta_{0}+2 \theta_{0}^{\prime}\right)$ on $[-m, m]$. We let $\kappa$ be a Lipschitz constant for $f_{0}$, and for any $\delta>0$ let $a \in\left(0, \theta_{0}\right)$ be such that if $w_{t}=w_{x x}$ and $w(0, \cdot) \geq \theta_{0} \chi_{(-m, m)}$, then $w(\delta, \cdot) \geq a e^{\kappa \delta} \chi_{(-m-M, m+M)}$. This means that whenever $u$ solves (1.1) with $f \geq f_{0}$ and $u\left(t^{\prime}, \cdot\right) \geq \theta_{0} \chi_{(A-m, A+m)}$ for some $A \in \mathbb{R}$, then

$$
u\left(t^{\prime}+\delta, \cdot\right) \geq a \chi(A-m-M, A+m+M) .
$$


Next, for any given $K<\infty$ we pick any (Lipschitz) even-in- $x$ pure bistable $f \geq f_{0}$ such that $f(x, u)=f_{0}(u)$ when $u \notin\left(\frac{a}{2}, p(x)\right)$ and

$$
f(x, u)=f_{0}(u)+K \operatorname{dist}\left(u,\left\{\frac{a}{2}, \frac{3 \theta_{0}+\theta_{0}^{\prime}}{4}\right\}\right)
$$

when $|x-n M| \leq m$ for some $n \in \mathbb{Z}$ and $u \in\left[\frac{a}{2}, \frac{1}{4}\left(3 \theta_{0}+\theta_{0}^{\prime}\right)\right]$. If $K$ is large enough, this can be done so that $f$ is indeed pure bistable. It is now clear from (4.1) that if $u$ solves (1.1) and $u\left(t^{\prime}, \cdot\right) \geq \theta_{0} \chi_{(n M-m, n M+m)}$ for some $n \in \mathbb{Z}$, then we have

$$
u\left(t^{\prime}+2 \delta, \cdot\right) \geq \theta_{0} \chi_{((n-1) M-m,(n+1) M+m)}
$$

provided $K$ is large enough. This immediately yields for such $K$ and $j=1,2, \ldots$,

$$
u\left(t^{\prime}+2 j \delta, \cdot\right) \geq \theta_{0} \chi_{((n-j) M-m,(n+j) M+m)} .
$$

We now pick any $\delta>0$ such that $4 \delta \sqrt{\kappa}<M$, then $K$ as above (so that (4.2) holds) and fix the corresponding $f$. If $u$ is any transition front for (1.1) (we only need to consider right-moving ones because $f$ is even in $x)$, we have $u(0, \cdot) \geq \theta_{0} \chi_{(n M-m, n M+m)}$ for some $n \in \mathbb{Z}$. From (4.2) we then get for $j=1,2, \ldots$,

$$
u(2 j \delta, \cdot) \geq \theta_{0} \chi_{((n-j) M-m,(n+j) M+m)} .
$$

On the other hand, we have

$$
u(t, x) \leq w(t, x):=p(x)+e^{-\sqrt{\kappa}(x-A-2 \sqrt{\kappa} t)}
$$

for some large $A<\infty$ and all $(t, x) \in \mathbb{R}^{+} \times \mathbb{R}$. This is true for $t=0$ because $u(0, \cdot)$ is bounded and $\lim _{x \rightarrow \infty} u(0, x)=0<P$, and then it holds for $t>0$ because $w$ is a super-solution of (1.1) (recall that $f(x, u)=f_{0}(u)$ for $u \geq p(x)$ and $\kappa$ is a Lipschitz constant for $f_{0}$ ):

$$
w_{t}-w_{x x}-f(x, w)=f_{0}(p(x))-f_{0}(w)+\kappa e^{-\sqrt{\kappa}(x-A-2 \sqrt{\kappa} t)} \geq 0 .
$$

This means that we have (in fact, for any solution of (1.1) with $\lim _{\sup } \operatorname{su}_{x \rightarrow \infty} u(0, x)<P$, and some large enough $A$ )

$$
u\left(t, 2 \sqrt{\kappa} t+A+\frac{1}{\kappa} \log \frac{2}{1-p(0)}\right) \leq \frac{1+p(0)}{2}
$$

This and (4.3) now show for $\varepsilon_{0}:=\min \left\{\theta_{0}, \frac{1-p(0)}{2}\right\}$ and $j=1,2, \ldots$ that $u(2 j \delta, \cdot)$ takes values within $\left[\varepsilon_{0}, 1-\varepsilon_{0}\right]$ on some interval of length

$$
(M-4 \delta \sqrt{\kappa}) j+n M-A-\frac{1}{\kappa} \log \frac{2}{1-p(0)} .
$$

Since $4 \delta \sqrt{\kappa}<M$, it follows that (1.1) with this pure bistable $f$ does not have any transition fronts (connecting 0 and 1 ).

The second claim is proved identically. Indeed, in the above argument we only needed that $u\left(t^{\prime}, \cdot\right) \geq \theta_{0} \chi_{(-m, m)}$ for some $t^{\prime} \in \mathbb{R}\left(\right.$ we can pick $\left.\theta_{0}=\frac{1}{2}\right)$, and $\lim _{\sup _{x \rightarrow \infty}} u\left(t^{\prime}, x\right)<P$. 


\section{Proof of Theorem 1.4(I)}

This proof is similar to the one of Theorem 1.2(i), with space-shifts replacing time-shifts at various points. Its existence part is slightly different, while the other two parts are essentially identical.

\section{Existence of a front}

We again let $u_{n}$ solve (1.2), but this time with initial condition $u_{n}(-n, x)=v(x)$ (where $\varepsilon_{0}, v$ are from the existence part of the proof of Theorem $\left.1.2(\mathrm{i})\right)$. We then let $\xi_{n}$ be maximal such that $u_{n}\left(0, \xi_{n}\right)=\frac{1}{2}$ (from $f \geq f_{0}$ we have $\lim _{n \rightarrow \infty} \xi_{n}=\infty$ ) and define $\tilde{u}_{n}(t, x):=$ $u_{n}\left(t, x+\xi_{n}\right)$. We again recover our candidate for a front $w$ (with $w(0,0)=\frac{1}{2}$ ) as a limit of a subsequence of these $\tilde{u}_{n}$, and it remains to prove (2.2) with $t \geq-n+T_{\varepsilon}$ instead of $t \geq T_{\varepsilon}$. Note that now $\left(u_{n}\right)_{x} \leq 0$, so this time $w_{x}<0$ will also follow.

We now pick $\zeta<\frac{c_{0}^{2}}{4}$ and $\theta_{1}^{\prime \prime}>\theta_{0}$ so that (2.3) holds, and again let $c_{\zeta}:=2 \sqrt{\zeta}$ and $c_{\xi}:=(\xi+\zeta) \zeta^{-1 / 2}$ (recall that $\left.\xi:=\max _{u \in(0,1]} \frac{f_{1}(u)}{u}\right)$. We then take for $t \geq-n$,

$$
\begin{gathered}
X_{n}(t):=\max \left\{x \in \mathbb{R} \mid u_{n}(s, x) \geq \theta_{1}^{\prime \prime} \text { for some } s \in[-n, t]\right\}, \\
Y_{n}(t):=\min \left\{y \in \mathbb{R} \mid u_{n}(s, x) \leq e^{-\sqrt{\zeta}(x-y)} \text { for all }(s, x) \in[-n, t] \times \mathbb{R}\right\} .
\end{gathered}
$$

The crucial lemma is now the following.

Lemma 5.1. (i) Lemma 2.1 (i) holds for any $n$ and $t \geq t^{\prime} \geq-n$.

(ii) For every $\varepsilon>0$ there is $r_{\varepsilon}<\infty$ such that for any $n$ and $t \geq t^{\prime} \geq-n$ we have

$$
\inf _{x \leq X_{n}\left(t^{\prime}\right)+c_{0}\left(t-t^{\prime}\right)-r_{\varepsilon}} u_{n}(t, x) \geq 1-\varepsilon .
$$

This $r_{\varepsilon}$ only depends on $\varepsilon, f_{0}, f_{1}$.

Proof. (i) This is identical to the proof of Lemma 2.1(i).

(ii) This is immediate from the spreading results in [2,11], $\theta_{1}^{\prime \prime}>\theta_{0}$, and $\left(u_{n}\right)_{x} \leq 0$ (here $r_{\varepsilon}$ depends on $\varepsilon, f_{0}, \theta_{1}^{\prime \prime}$, and the latter depends only on $f_{0}, f_{1}$.)

The rest of the existence proof carries over from Theorem $1.2(\mathrm{i})$, with $t \geq-n$ instead of $t \geq 0$, the constant $C$ in (2.8) only depending on $f_{0}, f_{1}$, and $Z_{n, \varepsilon}^{-}\left(t+T_{\varepsilon}\right) \geq X_{n}(t)$ (with $T_{\varepsilon}:=r_{\varepsilon} c_{0}^{-1}$ ) following directly from (5.3). In particular, $T_{\varepsilon}$ now only depends on $\varepsilon, f_{0}, f_{1}$, hence so does the upper bound on the left-hand side of (2.2). This means that for any fixed $f_{0}, f_{1}$, the limits in (1.5) are uniform in all $K$ and all $f$ satisfying $(\mathrm{H})$ with $x$ replaced by $t$.

We note that again we used neither $\theta>0$ nor $\theta_{1}>0$ so far, hence existence of fronts extends to mixed bistable-ignition-monostable reactions.

\section{Uniqueness of the front and convergence of typical solutions to it}

This is virtually identical to the same proof in Theorem 1.2(i), but with time-shifts of solutions replaced by space-shifts. The only changes are the following. The definitions of $X_{u}, Y_{u}, X_{w}, Y_{w}, Y_{w, h}$ are adjusted as in (15.1) and (15.2), while those of $Z_{u}, Z_{u, \varepsilon}^{-}, Z_{u, \varepsilon}^{+}, Z_{w}, Z_{w, \varepsilon}^{-}, Z_{w, \varepsilon}^{+}$ stay unchanged. Each " $w_{t}>0$ " is replaced by " $w_{x}<0$ ". Claims (2.17) are replaced by

$$
\text { if } \pm\left[w\left(t_{0}, \cdot-x_{0}\right)-u\left(t_{0}, \cdot\right)\right] \leq \delta \text {, then } \pm\left[w\left(t, \cdot-x_{0}\right)-u(t, \cdot)\right] \leq \varepsilon \text {. }
$$


In their proofs we can assume $x_{0}=0$ and use

$$
z_{ \pm}(t, x):=u\left(t, x \mp \frac{\varepsilon}{\Omega}\left(1-e^{-\sqrt{\zeta}\left(c_{0}-c_{\zeta}\right)\left(t-t_{0}\right) / 4}\right)\right) \pm b_{\varepsilon} e^{-\sqrt{\zeta}\left(x-Y_{w}\left(t_{0}\right)-c_{\zeta}\left(t-t_{0}\right)\right) / 2}
$$

(with $Y_{u}\left(t_{0}\right)$ instead of $Y_{w}\left(t_{0}\right)$ in the - case), where $\Omega$ is such that $\left|u_{x}\right| \leq \Omega$ for $t \geq 1$. The time-shift $\tau_{w}$ is replaced by the space-shift

$$
\xi_{w}:=\inf \left\{\xi \in \mathbb{R} \mid \liminf _{t \rightarrow \infty} \inf _{x \in \mathbb{R}}[w(t, x-\xi)-u(t, x)] \geq 0\right\},
$$

and $w\left(t+\tau_{w}, x\right)$ is replaced by $w\left(t, x-\xi_{w}\right)$ in the corresponding argument. In the last paragraph of the uniqueness proof we use initial conditions $u_{n}(-n, x)=v(x)$ and obtain for all $t^{\prime} \in \mathbb{R}, \varepsilon>0$, and some $\xi_{t^{\prime}, \varepsilon}$,

$$
\sup _{t \geq t^{\prime} \& x \in \mathbb{R}}\left|w_{1}(t, x)-w_{2}\left(t, x-\xi_{t^{\prime}, \varepsilon}\right)\right|<\varepsilon .
$$

This concludes the proof of uniqueness of the front (up to translation in $x$ ), and the claim of convergence of typical solutions to its space-shifts uses the same adjustments.

\section{Proof of Theorem 1.4(II)}

This is an immediate corollary of the following result, which is an analog of [34, Theorem 1.3] for time-dependent ignition reactions.

Theorem 6.1. Let $f$ be an ignition reaction, satisfying $(H)$ with each $x$ replaced by $t$, with $c_{0}$ the unique front speed for $f_{0}$. Assume that there are $\zeta<\frac{c_{0}^{2}}{4}$ and $\eta>0$ such that

$$
\inf _{t \in \mathbb{R} \& u \in\left[\alpha_{f}(t), \theta_{0}\right]} f(t, u) \geq \eta, \quad \text { where } \alpha_{f}(t):=\inf \{u \in(0,1) \mid f(t, u) \geq \zeta u\} .
$$

Then the claims in Theorem 1.2(i) hold for (1.2), with uniqueness of the front up to translations in $x$ and with $w_{x}<0$ instead of $w_{t}>0$.

As in Section 3, the above hypothesis automatically holds for all pure ignition reactions, with any $\zeta \in\left(0, \frac{c_{0}^{2}}{4}\right)$ and $\eta$ depending on $\zeta, \theta_{1}, K, \gamma$ (the latter from Definition 1.1). This proves Theorem 1.4(ii), so it remains to prove Theorem 6.1.

In fact, we only need to prove the existence part of the result. This is because the remaining claims are then proved identically to Theorem 1.4(i). Moreover, the beginning and the end of the proof of the existence part are also identical to that of Theorem 1.4(i). There is, however, a difference in Lemma 5.1(ii) because there need not be any $\theta_{1}^{\prime \prime}>\theta_{0}$ such that (2.3) holds. This will also require a slightly more refined part (i).

We use the notation from the beginning of the existence part of Section 5 (recall, in particular, that $\varepsilon_{0}$ only depends on $f_{0}$ ), but with (5.1) replaced by

$$
X_{n}(t):=\max \left\{x \in \mathbb{R} \mid u_{n}(t, x) \geq \alpha_{f}(t)\right\} .
$$

We will also need

$$
Z_{n}(t):=\max \left\{y \in \mathbb{R} \mid u_{n}(t, x) \geq 1-\varepsilon_{0} \text { for all } x<y\right\} .
$$

Notice that we have $Z_{n}(t) \leq X_{n}(t)$ due to $\alpha_{f}(t)<1-\varepsilon_{0}$ (see the argument just before the statement of Lemma 2.1). Here is the relevant version of Lemma 2.1, which also includes the analog of (2.8). 
Lemma 6.2. (i) For any $n$ and $t \geq t^{\prime} \geq-n$ we have (with $|A|$ the Lebesque measure of $A$ )

$$
Y_{n}(t)-Y_{n}\left(t^{\prime}\right) \leq c_{\zeta}\left(t-t^{\prime}\right)+\left(c_{\xi}-c_{\zeta}\right)\left|\left\{s \in\left[t^{\prime}, t\right] \mid X_{n}(s)>Y_{n}\left(t^{\prime}\right)\right\}\right|
$$

(ii) For every $\varepsilon>0$ there is $r_{\varepsilon}<\infty$ such that for any $n$ and $t \geq t^{\prime} \geq-n$ we have

$$
\inf _{x \leq Z_{n}\left(t^{\prime}\right)+c_{0}\left(t-t^{\prime}\right)-r_{\varepsilon}} u_{n}(t, x) \geq 1-\varepsilon .
$$

There is also $C$ such that

$$
\sup _{n \in \mathbb{N} \& t \geq-n}\left|Y_{n}(t)-Z_{n}(t)\right| \leq C .
$$

The $r_{\varepsilon}$ only depends on $\varepsilon, f_{0}$, while $C$ only depends on $f_{0}, f_{1}, K, \zeta, \eta$.

Lemma 6.2(ii) immediately yields $Z_{n, \varepsilon}^{-}\left(t+T_{\varepsilon}\right) \geq Z_{n}(t)$ (with $T_{\varepsilon}:=r_{\varepsilon} c_{0}^{-1}$ ), and as at the end of the proof of Theorem 1.2(i), we obtain an upper bound on the left-hand side of (2.2). This depends on $\varepsilon, f_{0}, f_{1}, K, \zeta, \eta$, so the limits in (1.5) depend on $f_{0}, f_{1}, K, \zeta, \eta$. Therefore, to finish the proof of Theorem 6.1, it remains to prove the lemma.

Proof. (i) Let $A:=\left\{s \geq t^{\prime} \mid X_{n}(s)>Y_{n}\left(t^{\prime}\right)\right\}$ and let $a(t):=c_{\zeta}\left(t-t^{\prime}\right)+\left(c_{\xi}-c_{\zeta}\right)\left|A \cap\left[t^{\prime}, t\right]\right|$ for any $t \geq t^{\prime}$. Then $w(t, x):=e^{-\sqrt{\zeta}\left(x-Y_{n}\left(t^{\prime}\right)-a(t)\right)}$ satisfies $w_{t}=w_{x x}+\left(\zeta+(\xi-\zeta) \chi_{A}(t)\right) w$, while $u_{n}$ is a sub-solution of this PDE on $\left(t^{\prime}, \infty\right) \times\left(Y_{n}\left(t^{\prime}\right), \infty\right)$ due to the definition of $X_{n}$ and $\alpha_{f}$. Since also $w \geq 1>u_{n}$ on $\left(t^{\prime}, \infty\right) \times\left(-\infty, Y_{n}\left(t^{\prime}\right)\right]$, we have $w \geq u_{n}$ on $\left[t^{\prime}, \infty\right) \times \mathbb{R}$, and the result follows.

(ii) The first claim follows as in Lemma 5.1(ii), so we only need to prove (6.6) (and only the inequality $Y_{n}(t)-Z_{n}(t) \leq C$ because the opposite one is obvious, with $\left.C=\zeta^{-1}\left|\ln \left(1-\varepsilon_{0}\right)\right|\right)$.

Let $\beta>0$ be the smallest positive number such that $f_{1}(\beta)=\zeta \beta$, so that $\alpha_{f}(t) \geq \beta$ for all $f$ from $(\mathrm{H})$. Let also $\eta^{\prime}>0$ be such that any $K$-Lipschitz function greater than $\eta$ on $\left[0, \theta_{0}\right]$ and greater than $f_{0}$ on $\left[\theta_{0}, 1\right]$ is greater than $\eta^{\prime}$ on $\left[0,1-\frac{\varepsilon_{0}}{2}\right]$. And let $\delta:=\frac{1}{2}\left(c_{0}-c_{\zeta}\right)\left(c_{\xi}-c_{\zeta}\right)^{-1}>0$.

We first claim that for any large enough $T<\infty$ there is $L_{T}<\infty$ (depending also on $\left.\varepsilon_{0}, \eta^{\prime}, \delta\right)$ such that the following holds. If $A \subseteq(0, T)$ satisfies $|A| \geq \delta T$ and $v_{t}=v_{x x}+h(t)$ on $(0, T) \times\left(0, L_{T}\right)$ with initial condition $v(0, \cdot) \equiv \beta$, boundary conditions $v_{x}(\cdot, 0) \equiv v\left(\cdot, L_{T}\right) \equiv 0$, and $h(t) \geq 0$ such that $h(t) \geq \eta^{\prime}$ for each $t \in A$ for which $v(t, 0) \leq 1-\frac{\varepsilon_{0}}{2}$, then $v\left(T^{\prime}, 0\right) \geq 1-\varepsilon_{0}$ for each $T^{\prime} \in[\sup A, T]$. Indeed, this follows for each $T \geq\left(1-\frac{\varepsilon_{0}}{2}-\beta\right)\left(\eta^{\prime} \delta\right)^{-1}$ from parabolic regularity and the fact that if $L_{T}$ is replaced by $\infty$, then $v$ is only a function of $t$ and we obviously have $v\left(T^{\prime}, \cdot\right) \geq 1-\frac{\varepsilon_{0}}{2}$ for each $T^{\prime} \in[\sup A, T]$.

This, $\left(u_{n}\right)_{x} \leq 0$, and the comparison principle now yield for each large enough $T$ that if $t^{\prime} \geq-n$ and the set $A:=\left\{t \in\left[t^{\prime}, t^{\prime}+T\right] \mid X_{n}(t)>Y_{n}\left(t^{\prime}\right)\right\}$ satisfies $|A| \geq \delta T$, then

$$
Z_{n}\left(t^{\prime}+T\right) \geq X_{n}(\inf A)-L_{T} \geq Y_{n}\left(t^{\prime}\right)-L_{T} .
$$

Let us now take $T \geq 2 r_{\varepsilon_{0}}\left(c_{0}-c_{\zeta}\right)^{-1}$, so that $c_{\zeta} T+\left(c_{\xi}-c_{\zeta}\right) \delta T \leq c_{0} T-r_{\varepsilon_{0}}$ (then $T$ and $L_{T}$ depend only on $\left.f_{0}, f_{1}, K, \zeta, \eta\right)$. We find using (6.4) and (6.5) that if $|A| \leq \delta T$, then

$$
Z_{n}\left(t^{\prime}+T\right)-Z_{n}\left(t^{\prime}\right) \geq Y_{n}\left(t^{\prime}+T\right)-Y_{n}\left(t^{\prime}\right) .
$$

From (6.7), (6.8), and $Y_{n}\left(t^{\prime}+T\right) \leq Y_{n}\left(t^{\prime}\right)+c_{\xi} T$ (which is due to (i)) we obtain for $j=0,1, \ldots$

$$
Y_{n}(-n+j T)-Z_{n}(-n+j T) \leq \max \left\{r, L_{T}+c_{\xi} T\right\}
$$


where $r>0$ is such that $v \equiv 1-\varepsilon_{0}$ on $(-\infty,-r]$ (so that $Y_{n}(-n)-Z_{n}(-n) \leq r$ for each $n$ ). We also have $Z_{n}(t) \geq Z_{n}\left(t^{\prime}\right)-r$ for $t \geq t^{\prime}$ because $\left(u_{n}\right)_{x} \leq 0$ and $v$ is a sub-solution of (1.2). This, (6.4), and (6.9) now yield

$$
Y_{n}(t)-Z_{n}(t) \leq L_{T}+2 c_{\xi} T+2 r
$$

for all $t \geq-n$, finishing the proof.

Note that again we used neither $\theta>0$ nor $\theta_{1}>0$ in the proof of the existence part of Theorem [6.1, so that result extends to mixed ignition-monostable reactions.

\section{Proof of Theorem 1.4(III)}

We will use the following lemma, in which we let

$$
\begin{aligned}
& g_{0}(u):= \begin{cases}0 & u \in\left[0, \frac{1}{2}\right], \\
\left(u-\frac{1}{2}\right)(1-u)\left(u-\frac{2}{3}\right) & u \in\left(\frac{1}{2}, 1\right],\end{cases} \\
& g_{1}(u):= \begin{cases}0 & u \in\left[0, \frac{1}{11}\right] \cup\left[\frac{1}{2}, \frac{2}{3}\right], \\
K \operatorname{dist}\left(u,\left\{\frac{1}{11}, \frac{1}{2}\right\}\right) & u \in\left(\frac{1}{11}, \frac{1}{2}\right) \\
\left(u-\frac{1}{2}\right)(1-u)\left(u-\frac{2}{3}\right) & u \in\left(\frac{2}{3}, 1\right],\end{cases}
\end{aligned}
$$

with some $K \geq 0$ (which will need to be large in (iv) below).

Lemma 7.1. There are $M>0$ and $a \in\left(0, \frac{1}{16}\right)$ such that the following hold.

(i) If $u_{t}=u_{x x}+g_{0}(u)$ on $(0,1) \times \mathbb{R}$ and $u(0, \cdot) \leq \chi_{(-\infty, 0]}+\frac{5}{8} \chi_{(0, \infty)}$, then

$$
u(1, \cdot) \leq \chi_{(-\infty, M]}+\left(\frac{5}{8}-2 a\right) \chi_{(M, \infty)} .
$$

(ii) If $u_{t}=u_{x x}+g_{1}(u)$ on $(1,4) \times \mathbb{R}$ and (7.1) holds, then for all $K \geq 0$,

$$
u(4, \cdot) \leq \chi_{(-\infty, 2 M]}+\left(\frac{5}{8}-a\right) \chi_{(2 M, \infty)} .
$$

(iii) If $u_{t}=u_{x x}+g_{0}(u)$ on $(-1,2) \times \mathbb{R}$ and $u(-1, \cdot) \geq \frac{4}{11} \chi_{(-M, M)}$, then

$$
\min \{u(0, \cdot), u(2, \cdot)\} \geq \frac{3}{11} \chi_{(-1,1)} .
$$

(iv) If $u_{t}=u_{x x}+g_{1}(u)$ on $(2,3) \times \mathbb{R}$ and $u(2, \cdot) \geq \frac{2}{11} \chi_{(-1,1)}$, then for all large enough $K$,

$$
u(3, \cdot) \geq \frac{5}{11} \chi_{(-4 M, 4 M)} .
$$

Proof. (i) This is obvious for any small enough $a>0$ and any large enough $M$ from $g_{0}\left(\frac{5}{8}\right)<0$. We fix this $a$, while $M$ may still be increased to satisfy (ii,iii).

(ii) This is obvious for the above $a$ and any large enough $M$ from $g_{1}\left(\frac{5}{8}-2 a\right)=0$.

(iii) This is obvious for any large enough $M$ from $g_{0}=0$ on $\left[0, \frac{1}{2}\right]$.

(iv) Fixing $M$ from (i,ii,iii), this follows for any large enough $K$ from $\frac{1}{11}<\frac{2}{11}<\frac{5}{11}<\frac{1}{2}$.

To prove Theorem 1.4(iii), we fix $a, M, K$ from the lemma and pick $\delta \in\left(0, \frac{a}{4}\right)$ (then $\left.e^{3 \delta}<1+a\right)$ and any pure bistable $(x, t)$-independent $f_{0} \leq f_{1}$ as in $(\mathrm{H})$ such that $f_{0} \leq g_{0}$ and

$$
\left|f_{j}(u)-g_{j}(u)\right| \leq \delta u
$$


for $u \in[0,1]$ and $j=0,1$ (note that such $f_{0}, f_{1}$ exist because $g_{0} \leq g_{1}$ and $\int_{0}^{1} g_{0}(u) d u>0$ ). We also let $f(t, u)$ be a pure bistable reaction satisfying $(\mathrm{H})$ with these $f_{0}, f_{1}$ and all $x$ replaced by $t$, which is time-periodic with period 4 and also satisfies

$$
f(t, \cdot)=f_{0} \text { for } t \in[0,1] \quad \text { and } \quad f(t, \cdot)=f_{1} \text { for } t \in[2,3] .
$$

If now $u$ solves (1.2) with $u\left(t^{\prime}, \cdot\right) \leq \chi_{\left(-\infty, x^{\prime}\right]}+\frac{5}{8} \chi_{\left(x^{\prime}, \infty\right)}$ for some $t^{\prime} \in 4 \mathbb{Z}$ and $x^{\prime} \in \mathbb{R}$, then Lemma 7.1(i) and $f_{0} \leq g_{0}$ show

$$
u\left(t^{\prime}+1, \cdot\right) \leq \chi_{\left(-\infty, x^{\prime}+M\right]}+\left(\frac{5}{8}-2 a\right) \chi_{\left(x^{\prime}+M, \infty\right)} .
$$

Then Lemma 7.1(ii), (7.2), and $e^{3 \delta}<1+a$ show

$$
u\left(t^{\prime}+4, \cdot\right) \leq \min \left\{1, e^{3 \delta}\left[\chi_{\left(-\infty, x^{\prime}+2 M\right]}+\left(\frac{5}{8}-a\right) \chi_{\left(x^{\prime}+2 M, \infty\right)}\right]\right\} \leq \chi_{\left(-\infty, x^{\prime}+2 M\right]}+\frac{5}{8} \chi_{\left(x^{\prime}+2 M, \infty\right)} .
$$

Iterating this, we obtain for $j=1,2, \ldots$,

$$
u\left(t^{\prime}+4 j, \cdot\right) \leq \chi_{\left(-\infty, x^{\prime}+2 j M\right]}+\frac{5}{8} \chi_{\left(x^{\prime}+2 j M, \infty\right)}
$$

Since $u(0, \cdot) \leq \chi_{(-\infty, A]}+\frac{5}{8} \chi_{(A, \infty)}$ for some $A \in \mathbb{R}$ whenever $\limsup _{x \rightarrow \infty} u(0, x)<\frac{5}{8}$, we obtain for any transition front $u$ for (1.2) (we only need to consider the right-moving ones because $f$ is $x$-independent) and some $A \in \mathbb{R}$,

$$
u(4 j, \cdot) \leq \chi_{(-\infty, A+2 j M]}+\frac{5}{8} \chi_{(A+2 j M, \infty)}
$$

for $j=1,2, \ldots$, an estimate analogous to (4.4).

Similarly, we can use Lemma 7.1(iii,iv), (7.2), and $e^{-3 \delta}>1-a>\frac{4}{5}$ to show that if $u$ solves (1.2) with $u\left(t^{\prime}-1, \cdot\right) \geq \frac{4}{11} \chi_{\left(x^{\prime}-M, x^{\prime}+M\right)}$ for some $t^{\prime} \in 4 \mathbb{Z}$ and $x^{\prime} \in \mathbb{R}$, then

$$
u\left(t^{\prime}+3, \cdot\right) \geq \frac{4}{11} \chi_{\left(x^{\prime}-4 M, x^{\prime}+4 M\right)} .
$$

Iteration then again yields for $j=1,2, \ldots$,

$$
u\left(t^{\prime}-1+4 j, \cdot\right) \geq \frac{4}{11} \chi\left(x^{\prime}-(3 j+1) M, x^{\prime}+(3 j+1) M\right),
$$

and one more application of Lemma 7.1 (iii), (17.2), and $e^{-3 \delta}>\frac{2}{3}$ yields for $j=1,2, \ldots$,

$$
u\left(t^{\prime}+4 j, \cdot\right) \geq \frac{2}{11} \chi_{\left(x^{\prime}-3 j M, x^{\prime}+3 j M\right)},
$$

Hence for any transition front $u$ and some $B \in \mathbb{R}$ we obtain

$$
u(4 j, \cdot) \geq \frac{2}{11} \chi_{(B-3 j M, B+3 j M)}
$$

for $j=1,2, \ldots$, an estimate analogous to (4.3).

This and (17.4) now show for $\varepsilon_{0}:=\frac{2}{11}$ and $j=1,2, \ldots$ that $u(4 j, \cdot)$ takes values within $\left[\varepsilon_{0}, 1-\varepsilon_{0}\right]$ on some interval of length $M j+B-A$. Since $M>0$, it follows that (1.2) with this pure bistable $f$ does not have any transition fronts (connecting 0 and 1 ).

Similarly to Section 4, the second claim is proved identically. 


\section{Proof of Corollary 1.5}

(i) This is immediate from uniqueness of the front and the fact that its single space/time period translate is also a transition front. We note that if $f_{1}^{\prime}(0)<0$ and $f_{0}^{\prime}(1)<0$, then the result also follows from our existence of transition fronts, $c_{0}>0$, and [7, Theorem 1.6].

(ii) The stationary ergodic assumption on $f$ means that there is a probability space $(\Omega, \mathcal{F}, \mathbb{P}), f: \Omega \rightarrow L_{\mathrm{loc}}^{\infty}(\mathbb{R} \times[0,1])$ is measurable and satisfies the required hypotheses uniformly in $\omega \in \Omega$, and there is a group $\left\{\pi_{k}\right\}_{k \in \mathbb{Z}}$ of measure preserving transformations acting ergodically on $\Omega$ such that either $f\left(\pi_{k} \omega ; x, u\right)=f(\omega ; x-k p, u)$ or $f\left(\pi_{k} \omega ; t, u\right)=f(\omega ; t-k p, u)$ for some $p>0$.

The proof of this part is similar to [34, Corollary 1.7]. Let us start with the spaceinhomogeneous reaction case. Let $v$ be the function from Section 2, and let $u_{m}$ solve (1.1) with initial condition $u_{m}(0, x):=v(x-m p)$ (so that $\left.\left(u_{m}\right)_{t}>0\right)$. For integers $n \geq m$ define

$$
\tau_{m, n}(\omega):=\inf \left\{t \geq 0 \mid u_{m}(t, x) \geq v(x-n p) \text { for all } x \in \mathbb{R}\right\} .
$$

As in [34], the subadditive ergodic theorem [14,17] applies to $\tau_{m, n}$ and yields finite positive deterministic limits

$$
\tau=\lim _{n \rightarrow \infty} \frac{\tau_{0, n}(\omega)}{n}=\lim _{n \rightarrow \infty} \frac{\tau_{-n, 0}(\omega)}{n}
$$

for almost all $\omega \in \Omega$. Uniform convergence (in $m$ and $\omega$ ) of the solution $u_{m}$ to the front $w_{\omega}$ in $L^{\infty}$ (see Remark 3 after Theorem 1.2) then shows that $c:=\frac{p}{\tau}$ is the asymptotic speed of $w_{\omega}$ as $|t| \rightarrow \infty$ for almost all $\omega \in \Omega$.

In the time-inhomogeneous reaction case we instead let $u_{m}$ solve (1.2) with initial condition $u_{m}(m p, x):=v(x)$, and for integers $n \geq m$ define

$$
\xi_{m, n}(\omega):=\sup \left\{y \in \mathbb{R} \mid u_{m}(n p, x) \geq v(x-y) \text { for all } x \in \mathbb{R}\right\} .
$$

This time the subadditive ergodic theorem yields finite positive deterministic limits

$$
\xi=\lim _{n \rightarrow \infty} \frac{\xi_{0, n}(\omega)}{n}=\lim _{n \rightarrow \infty} \frac{\xi_{-n, 0}(\omega)}{n}
$$

for almost all $\omega \in \Omega$, and it again follows that $c:=p \xi$ is the asymptotic speed of $w_{\omega}$ as $|t| \rightarrow \infty$ for almost all $\omega \in \Omega$.

\section{REFERENCES}

[1] N.D. Alikakos, P.W. Bates, and X. Chen, Periodic traveling waves and locating oscillating patterns in multidimensional domains, Trans. Amer. Math. Soc. 351 (1999), 2777-2805.

[2] D.G. Aronson and H.F. Weinberger, Nonlinear diffusion in population genetics, combustion, and nerve pulse propagation. Partial differential equations and related topics (Program, Tulane Univ., New Orleans, La., 1974), 5-49. Lecture Notes in Math., Vol. 446, Springer, Berlin, 1975.

[3] H. Berestycki, The influence of advection on the propagation of fronts in reaction-diffusion equations, Nonlinear PDEs in Condensed Matter and Reactive Flows, NATO Science Series C, 569, H. Berestycki and Y. Pomeau eds, Kluwer, Doordrecht, 2003.

[4] H. Berestycki and F. Hamel, Non-existence of travelling front solutions of some bistable reaction-diffusion equations, Adv. Diff. Equations 5 (2000), 723-746.

[5] H. Berestycki and F. Hamel, Front propagation in periodic excitable media, Comm. Pure and Appl. Math. 55 (2002), 949-1032.

[6] H. Berestycki and F. Hamel, Generalized transition waves and their properties, Comm. Pure Appl. Math 65 (2012), 592-648. 
[7] W. Ding, F. Hamel, and X.-Q. Zhao, Bistable pulsating fronts for reaction-diffusion equations in a periodic habitat, preprint.

[8] W. Ding, F. Hamel, and X.-Q. Zhao, Transition fronts for periodic bistable reaction-diffusion equations, preprint.

[9] A. Ducrot, T. Giletti, and H. Matano, Existence and convergence to a propagating terrace in onedimensional reaction-diffusion equations, Trans. Amer. Math. Soc. 366 (2014), 5541-5566.

[10] J. Fang and X.-Q. Zhao, Bistable traveling waves for monotone semiflows with applications, J. Europ. Math. Soc., to appear.

[11] P.C. Fife and J.B. McLeod, The approach of solutions of non-linear diffusion equations to traveling front solutions, Arch. Ration. Mech. Anal. 65 (1977), 335-361.

[12] P.C. Fife and L.A. Peletier, Clines Induced by Variable Selection and Migration Proc. R. Soc. Lond. B 214 (1981), 99-123.

[13] R. Fisher, The wave of advance of advantageous genes, Ann. Eugenics 7 (1937), 355-369.

[14] J.F.C. Kingman, The ergodic theory of subadditive stochastic processes, J. Roy. Statist. Soc. Sr. B 30 (1968), 499-510.

[15] A.N. Kolmogorov, I.G. Petrovskii and N.S. Piskunov, Étude de l'équation de la diffusion avec croissance de la quantité de matière et son application à un problème biologique, Bull. Moskov. Gos. Univ. Mat. Mekh. 1 (1937), 1-25.

[16] T.J. Lewis and J.P. Keener, Wave-block in excitable media due to regions of depressed excitability, SIAM J. Appl. Math. 61 (2000), 293-316.

[17] T.M. Liggett, An improved subadditive ergodic theorem, Ann. Probab. 13 (1985), 1279-1285.

[18] H. Matano, several conference talks.

[19] A. Mellet, J. Nolen, J.-M. Roquejoffre and L. Ryzhik, Stability of generalized transition fronts, Commun. PDE 34 (2009), 521-552.

[20] A. Mellet, J.-M. Roquejoffre and Y. Sire, Generalized fronts for one-dimensional reaction-diffusion equations, Discrete Contin. Dyn. Syst. 26 (2010), 303-312.

[21] J. Nolen, J.-M. Roquejoffre, L. Ryzhik, and A. Zlatoš, Existence and non-existence of Fisher-KPP transition fronts, Arch. Ration. Mech. Anal. 203 (2012), 217-246.

[22] J. Nolen and L. Ryzhik, Traveling waves in a one-dimensional heterogeneous medium, Ann. Inst. H. Poincaré Anal. Non Linéaire 26 (2009), 1021-1047.

[23] W. Shen, Traveling waves in time almost periodic structures governed by bistable nonlinearities, I. Stability and uniqueness, J. Diff. Equations 159 (1999), 1-54.

[24] W. Shen, Traveling waves in time almost periodic structures governed by bistable nonlinearities, II. Existence, J. Diff . Equations 159 (1999), 55-101.

[25] W. Shen, Traveling waves in diffusive random media, J. Dynam. Differ. Equat. 16 (2004), 1011-1060.

[26] W. Shen, Traveling waves in time dependent bistable equations. Differential Integral Equations 19 (2006), 241-278.

[27] W. Shen and Z. Shen, Transition fronts in time heterogeneous and random media of ignition type, preprint.

[28] W. Shen and Z. Shen, Stability, Uniqueness and Recurrence of Generalized Traveling Waves in Time Heterogeneous Media of Ignition Type, preprint.

[29] N. Shigesada, K. Kawasaki, and E. Teramoto, Traveling periodic waves in heterogeneous environments, Theor. Population Biol. 30 (1986), 143-160.

[30] S. Vakulenko and V. Volpert, Generalized travelling waves for perturbed monotone reaction-diffusion systems, Nonlinear Anal. 46 (2001), 757-776.

[31] J. Xin, Existence and nonexistence of travelling waves and reaction-diffusion front propagation in periodic media, J. Stat. Phys. 73 (1993), 893-926.

[32] J. Xin, Front propagation in heterogeneous media, SIAM Rev. 42 (2000), 161-230.

[33] J. Xin, An introduction to fronts in random media, Springer, New York, 2009.

[34] A. Zlatoš, Generalized traveling waves in disordered media: Existence, uniqueness, and stability, Arch. Ration. Mech. Anal. 208 (2013), 447-480.

[35] A. Zlatoš, Propagation of reaction in inhomogeneous media, preprint. 
Department of Mathematics, University of Wisconsin, Madison, Wi 53706, USA EMAIL:ZLATOS@MATH.WISC.EDU 